Article

\title{
Stability of an Axisymmetric Liquid Metal Flow Driven by a Multi-Pole Rotating Magnetic Field
}

\author{
Toshio Tagawa ${ }^{1, *}$ and Kewei Song ${ }^{2}$ \\ 1 Department of Aeronautics and Astronautics, Tokyo Metropolitan University, Hino 191-0065, Japan \\ 2 School of Mechanical Engineering, Lanzhou Jiaotong University, Lanzhou 730070, China; \\ songkw@mail.lzjtu.cn \\ * Correspondence: tagawa-toshio@tmu.ac.jp; Tel.: +81-42-585-8662
}

Received: 13 March 2019; Accepted: 18 April 2019; Published: 21 April 2019

\begin{abstract}
The stability of an electrically conducting fluid flow in a cylinder driven by a multi-pole rotating magnetic field is numerically studied. A time-averaged Lorentz force term including the electric potential is derived on the condition that the skin effect can be neglected and then it is incorporated into the Navier-Stokes equation as a body force term. The axisymmetric velocity profile of the basic flow for the case of an infinitely long cylinder depends on the number of pole-pairs and the Hartmann number. A set of linearized disturbance equations to obtain a neutral state was successfully solved using the highly simplified marker and cell (HSMAC) method together with a Newton-Raphson method. For various cases of the basic flow, depending on both the number of pole-pairs and the Hartmann number, the corresponding critical rotational Reynolds numbers for the onset of secondary flow were obtained instead of using the conventional magnetic Taylor number. The linear stability analyses reveal that the critical Reynolds number takes its minimum at a certain value of the Hartmann number. On the other hand, the velocity profile for cases of a finite length cylinder having a no-slip condition at the flat walls generates the Bödewadt boundary layers and such flows need to be computed including the non-linear terms of the Navier-Stokes equation.
\end{abstract}

Keywords: rotating magnetic field; magnetohydrodynamics; secondary flow; skin effect; Taylor-Görtler instability

\section{Introduction}

In material processing, such as semiconductor crystal growth or continuous casting of steel, applying a magnetic field is the most promising method to control molten metal flows. Among various types of magnetic field, the stationary magnetic field is used to damp out electrically conducting melt flow whereas the rotating magnetic field is considered to be useful to stir the melt flow due to the Lorentz force. The rotating magnetic field is a type of alternating magnetic field and its strength, field shape and angular frequency are the crucial parameters. Since there is a variety of rotating magnetic fields, the effect on the conducting melt flow also varied widely.

As a pioneering work, Moffat [1] investigated the effect of a rotating magnetic field on the electrically conducting fluid flow in a cylindrical vessel. He pointed out that the field strength and angular frequency are the two most important factors on the condition that the skin effect takes place. Richardson [2] analytically demonstrated the critical condition of the onset of secondary flow for an electrically conducting fluid in an infinitely long cylindrical enclosure. His analysis is limited to the case that the applied rotating magnetic field is weak enough to define the magnetic Taylor number and its angular frequency is slow enough to neglect the skin effect. Sneyd [3], Davidson and Hunt [4], and Davidson [5] also performed fundamental studies on the flow driven by a rotating magnetic field. 
In references [2-5], the common feature of the treatment of analysis is to neglect the skin effect and to use the time-averaged Lorentz force as a driving force in the azimuthal direction.

Concerning the application of the rotating magnetic field on the crystal growth, not only the fluid flow but also the temperature field and/or concentration field should be taken into account. Such studies can be found in references Barz et al. [6], Ghaddar et al. [7], Mößner and Gerbeth [8], Ben Hadid et al. [9], and Patzold et al. [10] and some of these include the discussion of the flow transition. Friedrich et al. [11] studied the effect of a rotating magnetic field on the Rayleigh-Bénard convection by using the magnetic Taylor number. Vizman et al. [12] demonstrated the three-dimensional simulation for a flow driven by a rotating magnetic field under the influence of buoyancy caused by the temperature difference. Volz and Mazuruk [13,14] investigated the influence of a high-frequency rotating magnetic field on the flow instability within a cylindrical enclosure heated from below with considering the skin effect. Grants and Gerbeth $[15,16]$ conducted stability analyses for both axisymmetric and three-dimensional flows driven by a rotating magnetic field in a cylindrical enclosure. Later, they also experimentally investigated the flow transition to turbulence [17].

The group of Martin and Witkowski studied the flow driven by an arbitrary frequency of the rotating magnetic field [18], and an asymmetric flow driven by a low frequency of the rotating magnetic field [19]. They also studied that a case of high frequency for a finite length of cylindrical enclosure [20], the influence of the rotating magnetic field on the instability of the thermo-capillary convection for the floating zone method [21], and the effect of a rotating magnetic field on a flow in a rotating cylindrical vessel [22]. Feonychev and Bodareva [23] considered the effect of a rotating magnetic field on the flow stability subjected to the gravitational force and/or surface tension. Nikrityuk et al. [24] carried out numerical computation for a flow driven by a rotating magnetic field in a cylindrical enclosure with various aspect ratios. Lyubimov et al. [25] performed linear stability analysis for a flow driven by a rotating magnetic field in a cylindrical enclosure whose axis is set horizontally and the temperature gradient is parallel to its axis.

The group of Ando and Ueno [26-30] studied the induction pump for a liquid metal using the rotating and twisted magnetic field experimentally, numerically and theoretically. The rotating twisted magnetic field, which can drive the molten liquid both in the azimuthal and axial directions, is generated by a stator with three pairs of helical windings. They carried out a molten metal circulation experiment using molten gallium and confirmed that the conventional slip-thrust relation is satisfied in the experiments [26-28]. Numerical simulation with the same condition performed in a previous experiment was carried out under the magnetic Stokes approximation for a small shielding parameter [29]. Asymptotic analysis [30] for a small magnetic Reynolds number clarifies that the temporal mean value of the axial driving force is almost uniform but rather small when the twist ratio is considerably smaller than 1 .

As mentioned above, the use of a rotating magnetic field is rather limited to the metal processes such as the control of the semiconductor melt and/or stirring and driving of the electrically conducting fluids. The most important point in such numerical analyses related to the rotating magnetic field is to model the Lorentz force which is responsible for the liquid metal flow. Most of the literature mentioned above assumed axisymmetric flow, neglecting the skin effect, low magnetic field, and time-averaged Lorentz force. In such a situation, the magnetic Taylor number is the only significant parameter that governs the fluid flow. However, recent development of superconducting magnets enables us to utilize a high magnetic field. Therefore, we aim to elucidate the fundamental mechanism of the flow instability caused by the strong rotating magnetic field in which the electric potential cannot be neglected. The present paper describes the modeling of the Lorentz force in which the influence of the number of pole-pairs is taken into account and carries out linear stability analysis of liquid metal driven by a multi-pole rotating magnetic field. 


\section{Configuration of Problem and Governing Equations}

\subsection{Problem Considered}

As shown in Figure 1, we consider a fundamental case that an electric conducting fluid filled in a cylindrical vessel is subject to an applied rotating magnetic field. The rotating magnetic field induces the electrical current density in the conducting fluid and it interacts with the magnetic field to generate the electromagnetic force. This phenomenon seems to be easily understood but it widely depends on both the angular velocity and the strength of the rotating magnetic field. We consider that the cylindrical vessel is stationary and, therefore, the boundary layer tends to become unstable due to the Taylor-Görlter instability in the vicinity of the side wall. The electrically conducting fluid considered is assumed to be an incompressible Newtonian fluid and the viscous dissipation and Joule heating are neglected in this analysis. The continuity and momentum equations are as follows:

$$
\begin{gathered}
\nabla \cdot \boldsymbol{u}=0 \\
\rho\left(\frac{\partial \boldsymbol{u}}{\partial t}+\boldsymbol{u} \cdot \nabla \boldsymbol{u}\right)=-\nabla p+\mu \nabla^{2} \boldsymbol{u}+\boldsymbol{f}
\end{gathered}
$$

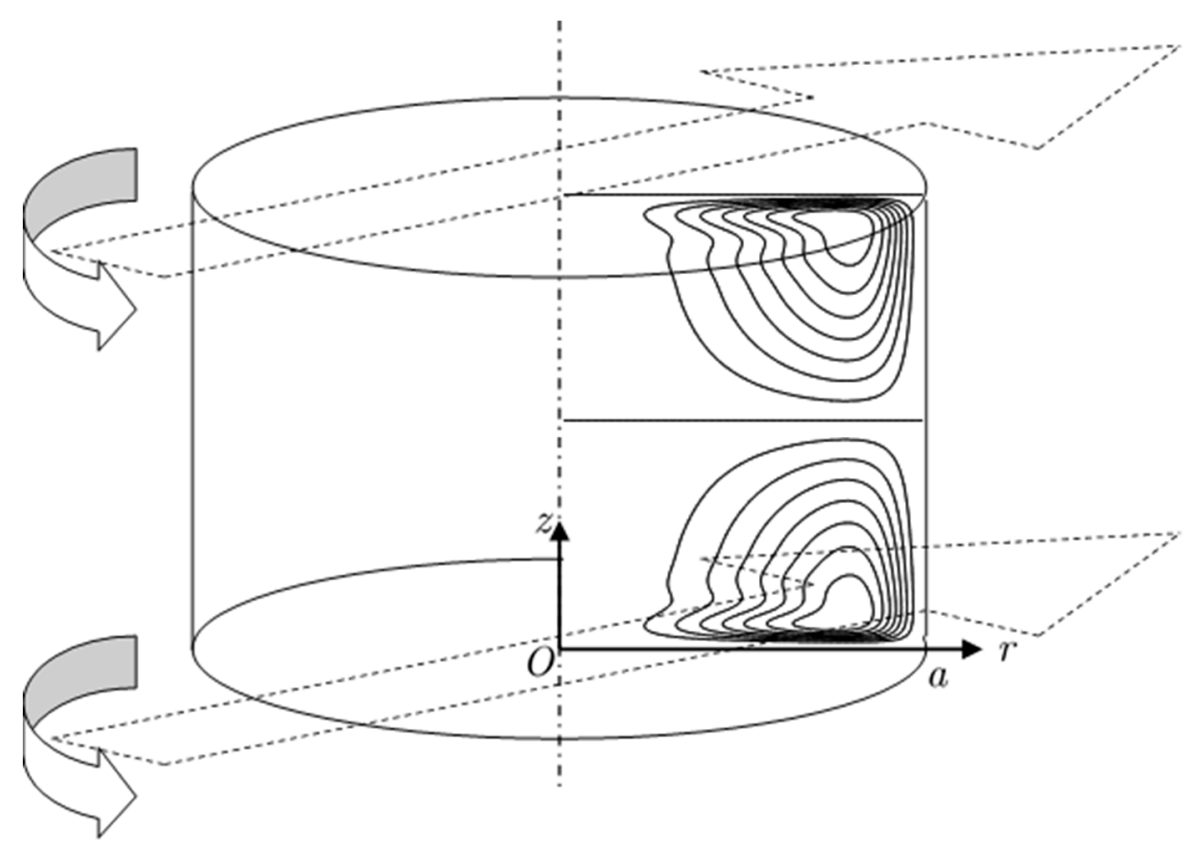

Figure 1. Schematic model for the problem. The arrows indicate the instantaneous direction of magnetic field, which rotates in a counterclockwise direction, for the case of $m=1$. The contour lines in a cross-section indicate an example of the meridional stream line.

The external force in the momentum equation is related to the electromagnetic fields, which are governed by the electromagnetic equations as follows:

$$
\begin{gathered}
\nabla \times \boldsymbol{E}=-\frac{\partial \boldsymbol{B}}{\partial t} \\
\nabla \cdot \boldsymbol{B}=0 \\
\nabla \times \boldsymbol{B}=\mu_{m} \boldsymbol{j} \\
\boldsymbol{j}=\sigma(\boldsymbol{E}+\boldsymbol{u} \times \boldsymbol{B}) \\
\nabla \cdot \boldsymbol{j}=0
\end{gathered}
$$




$$
f=j \times B
$$

Equation (3) is the Faraday's law, Equation (4) is the Gauss's law for magnetism, Equation (5) is the Ampère's law, Equation (6) is the Ohm's law, Equation (7) is the conservation of electric charge, and Equation (8) is the electromagnetic force. In order to satisfy Equation (4), a vector potential is introduced, as shown in Equation (9), together with the Coulomb gauge of Equation (10).

$$
\begin{gathered}
B=\nabla \times A \\
\nabla \cdot A=0
\end{gathered}
$$

From Equation (3), the electric field can be expressed with both the vector potential and scalar potential, as shown in Equation (11).

$$
E=-\nabla \phi-\frac{\partial A}{\partial t}
$$

\subsection{Formulation of Rotating Magnetic Field in Non-Conducting Media}

In this section, the formulation of a multi-pole rotating magnetic field in non-conducting media is considered. The rotating magnetic field satisfies both curl-free and divergence-free conditions at the place where the electric current density does not exist in non-conducting media. One of the possible vector potentials, which satisfies the Coulomb gauge, is expressed with a harmonic function.

$$
\nabla^{2} A=0
$$

In the polar coordinate system, a vector potential having only the axial component is considered herein.

$$
\frac{\partial^{2} A_{z}}{\partial r^{2}}+\frac{1}{r} \frac{\partial A_{z}}{\partial r}+\frac{1}{r^{2}} \frac{\partial^{2} A_{z}}{\partial \theta^{2}}=0
$$

We assume that the axial component of the vector potential can be divided into the amplitude function and the wave function rotating in the azimuthal direction as follows:

$$
A_{z}(r, \theta, t)=\tilde{A}_{z}(r) \cos (\omega t-m \theta)=\tilde{A}_{z}(r) \cos [m(\Omega t-\theta)]
$$

where, $m$ is the number of pole-pairs, $\omega$ is the angular frequency of the applied rotating magnetic field, and $\Omega$ is the angular velocity of the field. Equation (14) is substituted into Equation (13), and subsequently, we can obtain the Laplace equation regarding to the amplitude function.

$$
\left(\frac{d^{2}}{d r^{2}}+\frac{1}{r} \frac{d}{d r}-\frac{m^{2}}{r^{2}}\right) \tilde{A}_{z}=0
$$

Since we assume that the boundary condition for the magnetic field at the cylinder wall $(r=a)$ is $B_{0}$, the amplitude function of the vector potential is derived as follows:

$$
\tilde{A}_{z}(r)=\frac{B_{0} a}{m}\left(\frac{r}{a}\right)^{m}
$$

We obtain the axial component of vector potential.

$$
A_{z}(r, \theta, t)=\frac{B_{0} a}{m}\left(\frac{r}{a}\right)^{m} \cos (\omega t-m \theta)
$$

The radial and azimuthal components of the magnetic field can be derived. 


$$
\begin{gathered}
B_{r}(r, \theta, t)=\frac{1}{r} \frac{\partial A_{z}}{\partial \theta}=B_{0}\left(\frac{r}{a}\right)^{m-1} \sin (\omega t-m \theta), \\
B_{\theta}(r, \theta, t)=-\frac{\partial A_{z}}{\partial r}=-B_{0}\left(\frac{r}{a}\right)^{m-1} \cos (\omega t-m \theta)
\end{gathered}
$$

It is easily understood that these two equations satisfy the Laplace equations for the applied magnetic field as follows:

$$
\begin{aligned}
& \frac{\partial^{2} B_{r}}{\partial r^{2}}+\frac{1}{r} \frac{\partial B_{r}}{\partial r}-\frac{B_{r}}{r^{2}}+\frac{1}{r^{2}} \frac{\partial^{2} B_{r}}{\partial \theta^{2}}-\frac{2}{r^{2}} \frac{\partial B_{\theta}}{\partial \theta}=0, \\
& \frac{\partial^{2} B_{\theta}}{\partial r^{2}}+\frac{1}{r} \frac{\partial B_{\theta}}{\partial r}-\frac{B_{\theta}}{r^{2}}+\frac{1}{r^{2}} \frac{\partial^{2} B_{\theta}}{\partial \theta^{2}}+\frac{2}{r^{2}} \frac{\partial B_{r}}{\partial \theta}=0
\end{aligned}
$$

Figure 2 shows the magnetic field lines for several cases of the number of pole-pairs at a time instance. Equation (17) is used for visualization. Where, $\mathrm{N}$ and $\mathrm{S}$ indicate the magnetic poles. It can be recognized that the magnetic field does not tend to reach the core region as the number of pole-pairs is increased.

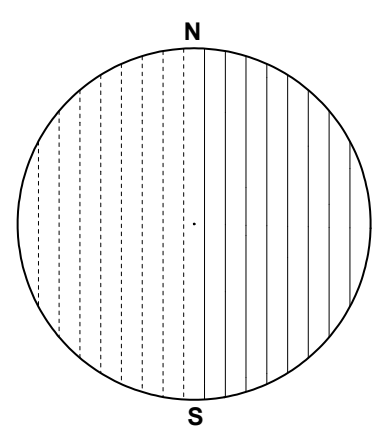

(a)

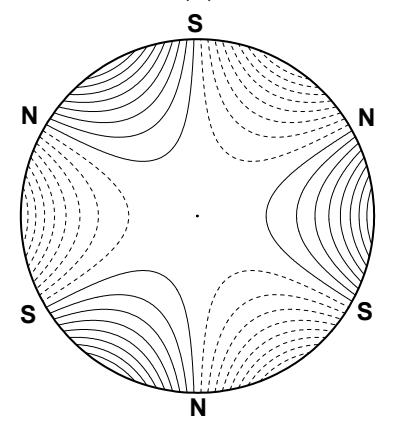

(c)

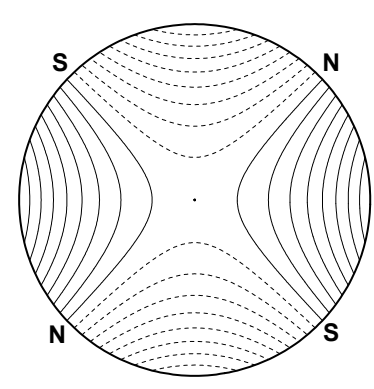

(b)

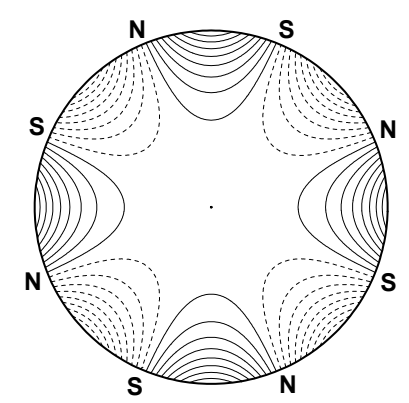

(d)

Figure 2. Magnetic field lines for four cases of number of pole-pairs. (a) $m=1$; (b) $m=2$; (c) $m=3$; (d) $m=4$. The solid and dotted lines are positive and negative values of $A_{\mathrm{z}}$ respectively.

\subsection{Formulation of Electromagnetic Force}

From Equations (5), (6) and (3), the induction equation can be derived.

$$
\frac{\partial \boldsymbol{B}}{\partial t}=\nabla \times(\boldsymbol{u} \times \boldsymbol{B})+\frac{1}{\sigma \mu_{m}} \nabla^{2} \boldsymbol{B}
$$

When this equation is expressed with a non-dimensional form, two non-dimensional numbers appear.

$$
R_{\omega} \frac{\partial \boldsymbol{B}^{*}}{\partial \tau}=\operatorname{Re}_{m} \nabla \times\left(\boldsymbol{u} \times \boldsymbol{B}^{*}\right)+\nabla^{2} \boldsymbol{B}^{*},\left(\text { where } R_{\omega}=\frac{\Omega a^{2}}{v_{m}}, R e_{m}=\frac{u_{0} a}{v_{m}}\right)
$$

where, $\Omega$ represents the angular velocity of the applied rotating magnetic field, $a$ is the radius of the cylinder, $v_{m}$ is the magnetic viscosity, $u_{0}$ is the characteristic velocity, $R_{\omega}$ is the shielding parameter, 
and $R e_{m}$ is the magnetic Reynolds number. In this paper, it is assumed that the two non-dimensional numbers are much smaller than unity.

$$
R e_{m}<R_{\omega}<<1
$$

Therefore, only the second term on the right-hand-side of Equation (21) is significant and the magnetic field even in the conducting media is assumed to be the same as that in a vacuum. Since the profiles of the vector potential and magnetic field have been obtained as shown in Equations (17) and (18), each component of the electric current density can be calculated using Ohm's law.

$$
\begin{gathered}
j_{r}(r, \theta, z, t)=\sigma\left[-\frac{\partial \phi}{\partial r}+w B_{0}\left(\frac{r}{a}\right)^{m-1} \cos (\omega t-m \theta)\right] \\
j_{\theta}(r, \theta, z, t)=\sigma\left[-\frac{1}{r} \frac{\partial \phi}{\partial \theta}+w B_{0}\left(\frac{r}{a}\right)^{m-1} \sin (\omega t-m \theta)\right] \\
j_{z}(r, \theta, z, t)=\sigma\left[-\frac{\partial \phi}{\partial z}+\left(\frac{r \omega}{m}-v\right) B_{0}\left(\frac{r}{a}\right)^{m-1} \sin (\omega t-m \theta)-u B_{0}\left(\frac{r}{a}\right)^{m-1} \cos (\omega t-m \theta)\right]
\end{gathered}
$$

The terms of electric potential have to be obtained so as to satisfy Equation (7) of the conservation of electric charge, and the following Poisson equation is derived.

$$
\begin{aligned}
& \frac{\partial^{2} \phi}{\partial r^{2}}+\frac{1}{r} \frac{\partial \phi}{\partial r}+\frac{1}{r^{2}} \frac{\partial^{2} \phi}{\partial \theta^{2}}+\frac{\partial^{2} \phi}{\partial z^{2}} \\
& =B_{0}\left(\frac{r}{a}\right)^{m-1}\left[\left(\frac{1}{r} \frac{\partial w}{\partial \theta}-\frac{\partial v}{\partial z}\right) \sin (\omega t-m \theta)+\left(\frac{\partial w}{\partial r}-\frac{\partial u}{\partial z}\right) \cos (\omega t-m \theta)\right]
\end{aligned}
$$

For the sake of simplicity in computation, we assume that the electric potential has the form as followed by Priede [31].

$$
\phi(r, \theta, z, t)=\phi_{1}(r, z, t) \sin (\omega t-m \theta)+\phi_{2}(r, z, t) \cos (\omega t-m \theta)
$$

Then, we have two Poisson equations, which do not include trigonometric functions.

$$
\begin{gathered}
\left(\frac{\partial^{2}}{\partial r^{2}}+\frac{1}{r} \frac{\partial}{\partial r}-\frac{m^{2}}{r^{2}}+\frac{\partial^{2}}{\partial z^{2}}\right) \phi_{1}=-B_{0}\left(\frac{r}{a}\right)^{m-1}\left(\frac{\partial v}{\partial z}\right) \\
\left(\frac{\partial^{2}}{\partial r^{2}}+\frac{1}{r} \frac{\partial}{\partial r}-\frac{m^{2}}{r^{2}}+\frac{\partial^{2}}{\partial z^{2}}\right) \phi_{2}=B_{0}\left(\frac{r}{a}\right)^{m-1}\left(\frac{\partial w}{\partial r}-\frac{\partial u}{\partial z}\right)
\end{gathered}
$$

where, the term of the azimuthal derivative of the axial component of the velocity is neglected in Equation (28) because of the assumption of axisymmetric flow in this study. By solving Equations (28) and (29), the electric potential that appeared in Ohm's law can be obtained. The electromagnetic force that appeared in Equation (8) is represented as shown in the next equation.

$$
f=\left(\begin{array}{l}
f_{r} \\
f_{\theta} \\
f_{z}
\end{array}\right)=\sigma B_{0}\left(\frac{r}{a}\right)^{m-1}\left(\begin{array}{l}
-\frac{\partial}{\partial z}\left[\phi_{1} \frac{\sin 2 \beta}{2}+\phi_{2} \frac{1+\cos 2 \beta}{2}\right]+\left(\frac{r \omega}{m}-v\right) B_{0}\left(\frac{r}{a}\right)^{m-1} \frac{\sin 2 \beta}{2}-u B_{0}\left(\frac{r}{a}\right)^{m-1} \frac{1+\cos 2 \beta}{2} \\
-\frac{\partial}{\partial z}\left[\phi_{1} \frac{1-\cos 2 \beta}{2}+\phi_{2} \frac{\sin 2 \beta}{2}\right]+\left(\frac{r \omega}{m}-v\right) B_{0}\left(\frac{r}{a}\right)^{m-1} \frac{1-\cos 2 \beta}{2}-u B_{0}\left(\frac{r}{a}\right)^{m-1} \frac{\sin 2 \beta}{2} \\
\frac{\partial}{\partial r}\left[\phi_{1} \frac{\sin 2 \beta}{2}+\phi_{2} \frac{1+\cos 2 \beta}{2}\right]+\frac{m}{r}\left[-\phi_{1} \frac{\sin 2 \beta}{2}+\phi_{2} \frac{1-\cos 2 \beta}{2}\right]-w B_{0}\left(\frac{r}{a}\right)^{m-1}
\end{array}\right)
$$

where, $\beta=\omega t-m \theta$ in Equation (30). As indicated in Equation (30), the electromagnetic force can be divided into the periodic oscillatory part and non-periodic averaged part. In this paper, the double frequency of the wave motion in the electromagnetic force is ignored. The non-periodic averaged force is arranged as follows. 


$$
f_{\text {ave }}=\left(\begin{array}{c}
\left\langle f_{r}\right\rangle \\
\left\langle f_{\theta}\right\rangle \\
\left\langle f_{z}\right\rangle
\end{array}\right) \cong \frac{\sigma B_{0}}{2}\left(\frac{r}{a}\right)^{m-1}\left(\begin{array}{c}
-\frac{\partial \phi_{2}}{\partial z}-u B_{0}\left(\frac{r}{a}\right)^{m-1} \\
-\frac{\partial \phi_{1}}{\partial z}+\left(\frac{r \omega}{m}-v\right) B_{0}\left(\frac{r}{a}\right)^{m-1} \\
\frac{1}{r^{m}} \frac{\partial}{\partial r}\left(r^{m} \phi_{2}\right)-2 w B_{0}\left(\frac{r}{a}\right)^{m-1}
\end{array}\right)
$$

This Lorentz force model derived here is coincident to that developed originally by Priede [31-33]. In this paper, the axisymmetric flow driven by the averaged force is considered. By combining Equations (1), (2) and (31), the Navier-Stokes equations, including the electromagnetic force in the cylindrical coordinate, are as follows:

$$
\begin{gathered}
\frac{\partial u}{\partial r}+\frac{u}{r}+\frac{\partial w}{\partial z}=0 \\
\frac{\partial u}{\partial t}+u \frac{\partial u}{\partial r}+w \frac{\partial u}{\partial z}-\frac{v^{2}}{r}=-\frac{1}{\rho} \frac{\partial p}{\partial r}+v\left(\frac{\partial^{2}}{\partial r^{2}}+\frac{1}{r} \frac{\partial}{\partial r}-\frac{1}{r^{2}}+\frac{\partial^{2}}{\partial z^{2}}\right) u+\frac{\sigma B_{0}}{2 \rho}\left(\frac{r}{a}\right)^{m-1}\left[-\frac{\partial \phi_{2}}{\partial z}-u B_{0}\left(\frac{r}{a}\right)^{m-1}\right] \\
\frac{\partial v}{\partial t}+u \frac{\partial v}{\partial r}+w \frac{\partial v}{\partial z}+\frac{u v}{r}=v\left(\frac{\partial^{2}}{\partial r^{2}}+\frac{1}{r} \frac{\partial}{\partial r}-\frac{1}{r^{2}}+\frac{\partial^{2}}{\partial z^{2}}\right) v+\frac{\sigma B_{0}}{2 \rho}\left(\frac{r}{a}\right)^{m-1}\left[-\frac{\partial \phi_{1}}{\partial z}+\left(\frac{r \omega}{m}-v\right) B_{0}\left(\frac{r}{a}\right)^{m-1}\right] \\
\frac{\partial w}{\partial t}+u \frac{\partial w}{\partial r}+w \frac{\partial w}{\partial z}=-\frac{1}{\rho} \frac{\partial p}{\partial z}+v\left(\frac{\partial^{2}}{\partial r^{2}}+\frac{1}{r} \frac{\partial}{\partial r}+\frac{\partial^{2}}{\partial z^{2}}\right) w+\frac{\sigma B_{0}}{2 \rho}\left(\frac{r}{a}\right)^{m-1}\left[\frac{1}{r^{m}} \frac{\partial}{\partial r}\left(r^{m} \phi_{2}\right)-2 w B_{0}\left(\frac{r}{a}\right)^{m-1}\right]
\end{gathered}
$$

\section{Linear Stability Analyses}

In this section, electrically conducting fluid flow driven by the multi-pole rotating magnetic fields in an infinitely long cylinder with the radius $a$ is considered.

\subsection{Basic Flows}

When the influence of the upper and lower ends is neglected, the fluid flow has only the azimuthal velocity and no electric field is generated. Since the inertial term and the electric potential term in Equation (34) disappear, it represents the balance between the electromagnetic and viscous forces.

$$
\left(\frac{d^{2}}{d r^{2}}+\frac{1}{r} \frac{d}{d r}-\frac{1}{r^{2}}\right) \bar{v}(r)+\frac{\sigma B_{0}^{2}}{2 \rho v}\left(\frac{r}{a}\right)^{2(m-1)}\left(\frac{r \omega}{m}-\bar{v}(r)\right)=0
$$

The solution of the basic flow can be obtained by solving the above equation. After making the equation non-dimensional, we get:

$$
\left(\frac{d^{2}}{d R^{2}}+\frac{1}{R} \frac{d}{d R}-\frac{1}{R^{2}}-\frac{H a^{2}}{2} R^{2(m-1)}\right) \bar{V}(R)=-\frac{H a^{2}}{2} R^{2 m-1} .
$$

Here, the dimensionless azimuthal velocity, dimensionless radius and the Hartmann number are defined as follows:

$$
\bar{V}(R)=\frac{\bar{v}(r)}{a \Omega}=\frac{\bar{v}(r)}{a \omega / m}, R=\frac{r}{a}, H a=B_{0} a \sqrt{\frac{\sigma}{\rho v}}
$$

The solution to Equation (37) with the no-slip boundary condition at the cylinder wall can be found using the modified Bessel function of the first kind as follows [34]:

$$
\bar{V}(R)=R-I_{1 / m}\left(\frac{H a R^{m}}{\sqrt{2} m}\right) / I_{1 / m}\left(\frac{H a}{\sqrt{2} m}\right), \text { where } I_{v}(x)=\sum_{n=0}^{\infty} \frac{x^{2 n+v}}{2^{2 n+v} n ! \Gamma(n+v+1)}
$$

Figure 3 shows the azimuthal velocity of the basic flow for several cases of the number of pole-pairs $(m)$ and the Hartmann number $(\mathrm{Ha})$. For a given Hartmann number, the azimuthal velocity decreases as 
the number of pole-pairs increases. For a given number of pole-pairs, the azimuthal velocity increases as the Hartmann number increases. For an extreme case of $m=1$ and $\mathrm{Ha}=32$, the core velocity attains the rigid-body rotation. This means that the core flow rotates with the same angular velocity as that of the applied rotating magnetic field. Figure 4 shows the azimuthal velocity gradient of the core flow at the axis of the cylinder for the three different cases of the number of pole-pairs. The azimuthal velocity gradient at the axis is proportional to the square of the Hartmann number for the cases of $H a<<1$. The azimuthal velocity gradient at $m=1$ is always larger than that at $m=2$ or 3 . This means that the rotating magnetic field with $m=1$ is more efficient to drive the azimuthal flow than that with $m=2$ or 3.

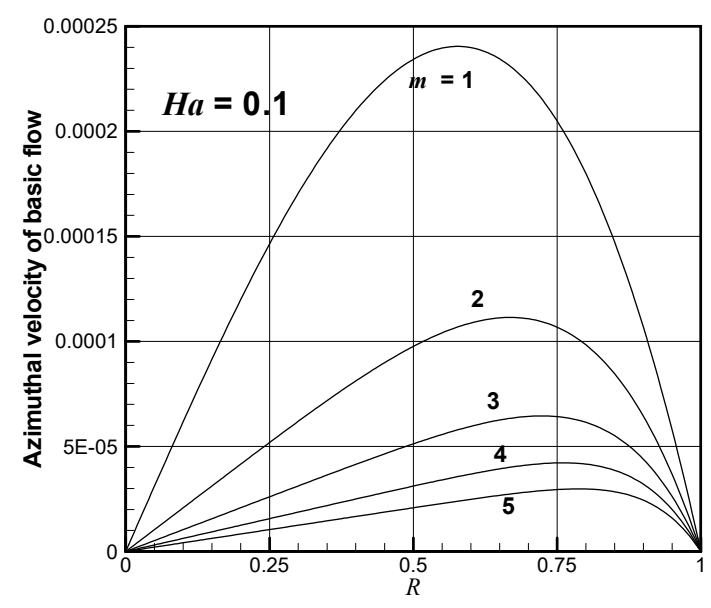

(a)

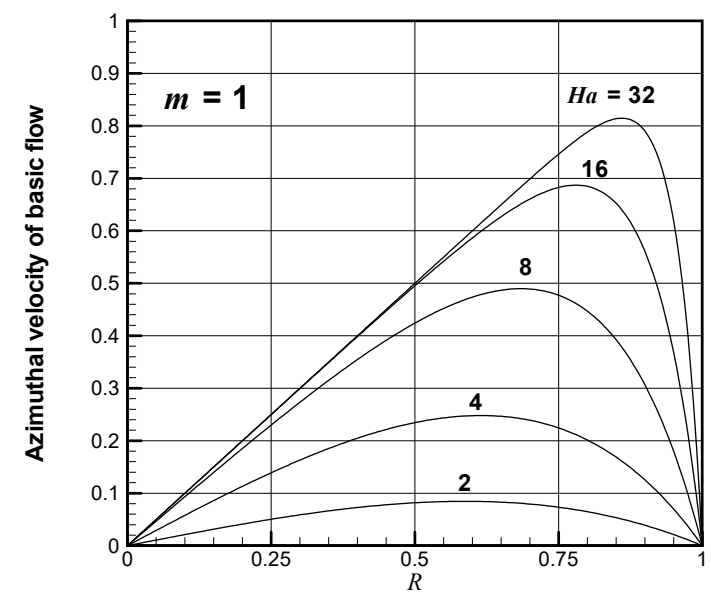

(c)

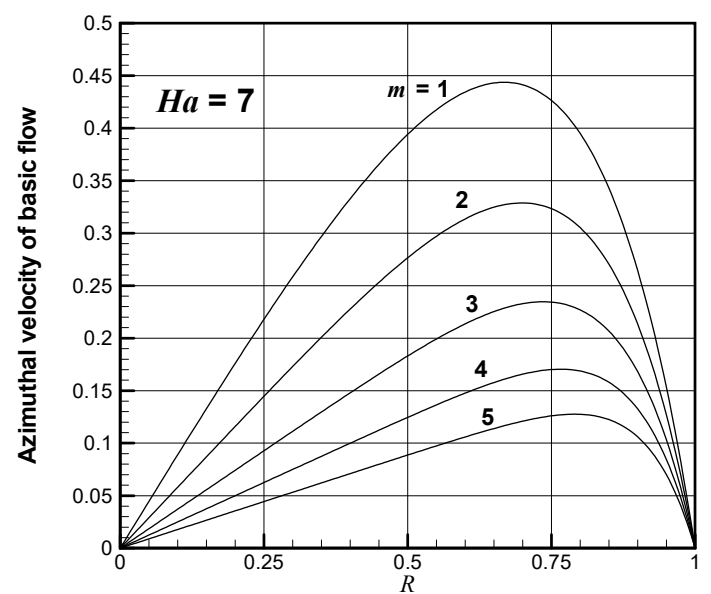

(b)

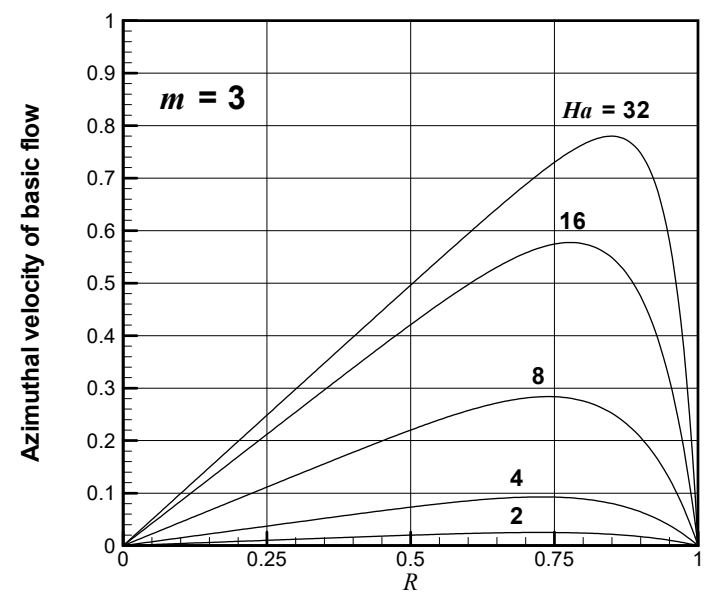

(d)

Figure 3. The azimuthal velocity of the basic flow for several numbers of the pole pairs and the Hartmann numbers. (a) $H a=0.1$; (b) $H a=7$; (c) $m=1$; (d) $m=3$. 


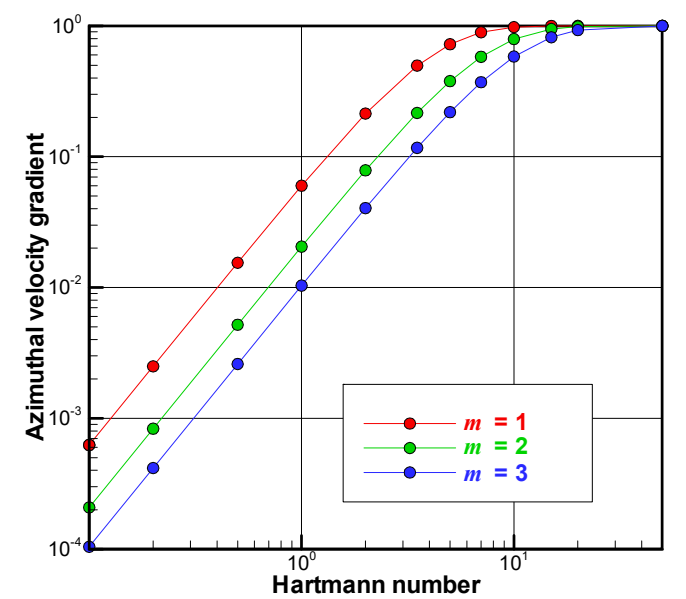

Figure 4. The azimuthal velocity gradient of the basic flow at the axis of cylinder for the three numbers of the pole-pairs.

\subsection{Disturbance Equations}

The basic velocity is not always realized, but it tends to become unstable for the higher values of the rotational Reynolds number. The present boundary layer flow along the concave surface is subjected to centrifugal instability. Therefore, toroidal vortices aligning along the axial direction are formed within the boundary layer near the sidewall. We assume that the perturbed components are axisymmetric and are superposed with the basic state as follows:

$$
\begin{aligned}
& u=u^{\prime}(r, z, t), v=\bar{v}(r)+v^{\prime}(r, z, t), w=w^{\prime}(r, z, t), p=\bar{p}(r)+p^{\prime}(r, z, t), \\
& \phi_{1}=\phi_{1}{ }^{\prime}(r, z, t), \phi_{2}=\phi_{2}{ }^{\prime}(r, z, t)
\end{aligned}
$$

where the basic velocity and pressure are expressed as follows:

$$
\begin{aligned}
& \bar{v}(r)=\Omega\left[r-a I_{1 / m}\left(\sqrt{\frac{\sigma}{2 \rho v}} \frac{B_{0} a}{m}\left(\frac{r}{a}\right)^{m}\right) / I_{1 / m}\left(\sqrt{\frac{\sigma}{2 \rho v}} \frac{B_{0} a}{m}\right)\right], \\
& \bar{p}(r)=\int \frac{\rho \bar{v}^{2}}{r} d r+\text { const. }
\end{aligned}
$$

Then, we can obtain the linearized perturbation equations and boundary conditions as follows:

$$
\begin{gathered}
\frac{\partial u^{\prime}}{\partial r}+\frac{u^{\prime}}{r}+\frac{\partial w^{\prime}}{\partial z}=0 \\
\frac{\partial u^{\prime}}{\partial t}=-\frac{1}{\rho} \frac{\partial p^{\prime}}{\partial r}+v\left(\frac{\partial^{2}}{\partial r^{2}}+\frac{1}{r} \frac{\partial}{\partial r}-\frac{1}{r^{2}}+\frac{\partial^{2}}{\partial z^{2}}\right) u^{\prime}+\frac{2 \bar{v}}{r} v^{\prime}+\frac{\sigma B_{0}}{2 \rho}\left(\frac{r}{a}\right)^{m-1}\left[-\frac{\partial \phi_{2}{ }^{\prime}}{\partial z}-u^{\prime} B_{0}\left(\frac{r}{a}\right)^{m-1}\right] \\
\frac{\partial v^{\prime}}{\partial t}=v\left(\frac{\partial^{2}}{\partial r^{2}}+\frac{1}{r} \frac{\partial}{\partial r}-\frac{1}{r^{2}}+\frac{\partial^{2}}{\partial z^{2}}\right) v^{\prime}-\left(\frac{d \bar{v}}{d r}+\frac{\bar{v}}{r}\right) u^{\prime}+\frac{\sigma B_{0}}{2 \rho}\left(\frac{r}{a}\right)^{m-1}\left[-\frac{\partial \phi_{1}{ }^{\prime}}{\partial z}-v^{\prime} B_{0}\left(\frac{r}{a}\right)^{m-1}\right] \\
\frac{\partial w^{\prime}}{\partial t}=-\frac{1}{\rho} \frac{\partial p^{\prime}}{\partial z}+v\left(\frac{\partial^{2}}{\partial r^{2}}+\frac{1}{r} \frac{\partial}{\partial r}+\frac{\partial^{2}}{\partial z^{2}}\right) w^{\prime}+\frac{\sigma B_{0}}{2 \rho}\left(\frac{r}{a}\right)^{m-1}\left[\frac{1}{r^{m}} \frac{\partial}{\partial r}\left(r^{m} \phi_{2}{ }^{\prime}\right)-2 w^{\prime} B_{0}\left(\frac{r}{a}\right)^{m-1}\right] \\
\left(\frac{\partial^{2}}{\partial r^{2}}+\frac{1}{r} \frac{\partial}{\partial r}-\frac{m^{2}}{r^{2}}+\frac{\partial^{2}}{\partial z^{2}}\right) \phi_{1}{ }^{\prime}=-B_{0}\left(\frac{r}{a}\right)^{m-1}\left(\frac{\partial v^{\prime}}{\partial z}\right) \\
\left(\frac{\partial^{2}}{\partial r^{2}}+\frac{1}{r} \frac{\partial}{\partial r}-\frac{m^{2}}{r^{2}}+\frac{\partial^{2}}{\partial z^{2}}\right) \phi_{2}{ }^{\prime}=B_{0}\left(\frac{r}{a}\right)^{m-1}\left(\frac{\partial w^{\prime}}{\partial r}-\frac{\partial u^{\prime}}{\partial z}\right)
\end{gathered}
$$

The boundary conditions for the perturbed components are given as follows: 


$$
\left\{\begin{array}{l}
r=0: u^{\prime}=0, v^{\prime}=0, \partial w^{\prime} / \partial r=0, \phi_{1}{ }^{\prime}=0, \phi_{2}{ }^{\prime}=0 \\
r=a: u^{\prime}=0, v^{\prime}=0, w^{\prime}=0, \partial \phi_{1}{ }^{\prime} / \partial r=0, \partial \phi_{2}{ }^{\prime} / \partial r=0
\end{array}\right.
$$

The normal modes of the form are as follows:

$$
\begin{aligned}
& u^{\prime}=\tilde{u}(r) \exp (\mathrm{i} \alpha z+s t), v^{\prime}=\tilde{v}(r) \exp (\mathrm{i} \alpha z+s t), w^{\prime}=\tilde{w}(r) \exp (\mathrm{i} \alpha z+s t), \\
& p^{\prime}=\tilde{p}(r) \exp (\mathrm{i} \alpha z+s t), \phi_{1}{ }^{\prime}=\tilde{\phi}_{1}(r) \exp (\mathrm{i} \alpha z+s t), \phi_{2}{ }^{\prime}=\tilde{\phi}_{2}(r) \exp (\mathrm{i} \alpha z+s t)
\end{aligned}
$$

where, $\alpha$ is the axial wavenumber which is real number, and $s$ is in general the complex number. In this study, $s$ is assumed to be a real number and is regarded as the growth rate of the disturbance since the stationary state at the onset of secondary flow is focused. If $s>0$, the basic flow is unstable enough to generate the secondary flow. If $s<0$, the basic flow is stable since the disturbance attenuates as time evolves. Using Equation (49), we can obtain ordinary differential equations for a neutral state of the onset of secondary flow in a dimensionless form as follows:

$$
\begin{gathered}
D_{*} \tilde{U}+\mathrm{i} k \tilde{W}=0 \\
\left(D D_{*}-k^{2}-H a^{2} R^{2(m-1)} / 2\right) \tilde{U}-D \tilde{P}+\operatorname{Re}(2 \bar{V} / R) \tilde{V}-\left(H a^{2} / 2\right) R^{m-1}\left(\mathrm{i} k \tilde{\Phi}_{2}\right)=0 \\
\left(D D_{*}-k^{2}-H a^{2} R^{2(m-1)} / 2\right) \tilde{V}-\operatorname{Re}\left(D_{*} \bar{V}\right) \tilde{U}-\left(H a^{2} / 2\right) R^{m-1}\left(\mathrm{i} k \tilde{\Phi}_{1}\right)=0 \\
\left(D_{*} D-k^{2}-H a^{2} R^{2(m-1)}\right) \tilde{W}-\mathrm{i} k \tilde{P}+\left(H a^{2} / 2\right) R^{m-1}(D+m / R) \tilde{\Phi}_{2}=0 \\
\left(D_{*} D-m^{2} / R^{2}-k^{2}\right) \tilde{\Phi}_{1}+R^{m-1}(\mathrm{i} k \tilde{V})=0 \\
\left(D_{*} D-m^{2} / R^{2}-k^{2}\right) \tilde{\Phi}_{2}-R^{m-1}(D \tilde{W}-\mathrm{i} k \tilde{U})=0
\end{gathered}
$$

where, $k$ is the dimensionless axial wavenumber, $R e$ is the rotational Reynolds number based on the angular velocity of the rotating magnetic field, and the dimensionless dependent variables such as velocity, pressure and electric potentials are as follows.

$$
\begin{aligned}
& R=\frac{r}{a}, H a=B_{0} a \sqrt{\frac{\sigma}{\rho v}}, k=\alpha a, \operatorname{Re}=\frac{\Omega a^{2}}{v} \\
& (\bar{V}, \tilde{U}, \tilde{V}, \tilde{W})=\frac{(\bar{v}, \tilde{u}, \tilde{v}, \tilde{w})}{\Omega a}, \tilde{P}=\frac{\tilde{p}}{\rho \Omega^{2} a^{2}},\left(\tilde{\Phi}_{1}, \tilde{\Phi}_{2}\right)=\frac{\left(\tilde{\phi}_{1}, \tilde{\phi}_{2}\right)}{\Omega a^{2} B_{0}}
\end{aligned}
$$

where, $D$ and $D_{*}$ represent the differential operators, which are defined as $D=d / d R$ and $D_{*}=d / d R+$ $1 / R$ respectively. The non-dimensional boundary conditions for the amplitude functions are as follows:

$$
\begin{cases}R=0: & \tilde{U}=0, \tilde{V}=0, D \tilde{W}=0, \tilde{\Phi}_{1}=0, \tilde{\Phi}_{2}=0 \\ R=1: & \tilde{U}=0, \tilde{V}=0, \tilde{W}=0, D \tilde{\Phi}_{1}=0, D \tilde{\Phi}_{2}=0\end{cases}
$$

For a given wavenumber and Hartmann number, the simultaneous ordinary equations are numerically solved together with the above boundary conditions to obtain the neutral Reynolds number at which the basic flow becomes unstable.

\subsection{Numerical Strategy}

The simultaneous ordinary Equations (50) to (55) were decomposed into their real and imaginary parts, respectively, and discretized on a one-dimensional equidistant staggered grid system, as shown in Figure 5. All the equations are discretized by the fourth order central difference method. The radial velocity is defined at cell boundaries, while the other variables such as the azimuthal and axial components of velocity and the pressure are defined at cell centers so as to satisfy the equation of continuity. The pressure is obtained by the HSMAC method [35]. The computational procedure is as 
follows. First, for a given number of pole-pairs $m$ and Hartmann number $H a$, the basic flow profile such as the azimuthal velocity and the pressure is numerically obtained by solving Equation (37). Then, for a given wavenumber $k$, its corresponding neutral Reynolds number can be obtained by using the Newton-Raphson method with Equations (50)-(55) during in which normalization of the amplitude functions is implemented in order to set them uniquely. In this study, a component of velocity at a certain point is fixed at unity during the computation. Such procedures are repeated by changing the value of $k$. Among the various values of $k$, the smallest value of the neutral Reynolds number is the critical Reynolds number.

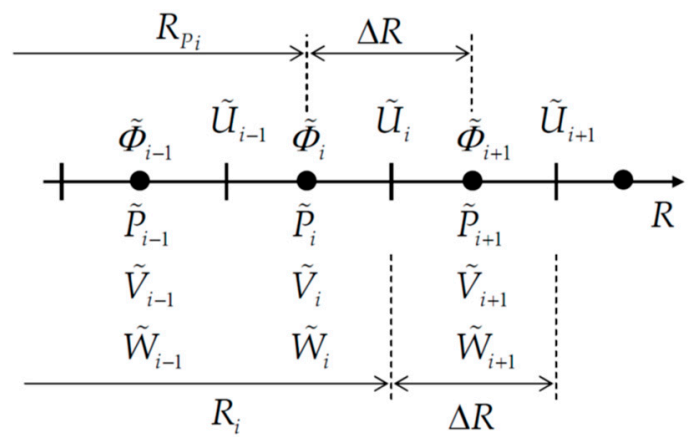

Figure 5. The one-dimensional equidistant staggered grid system for analyzing the disturbance equations.

\subsection{Results}

Table 1 shows the critical Reynolds numbers obtained for the various values of Hartmann number $(\mathrm{Ha})$ and number of pole-pairs $(\mathrm{m})$. The number of grids for all the analyses is 201, although the boundary layer formed in the vicinity of the side wall tends to be thin the Hartmann number increases. The dependency of the number of grids is quite small. For example, the critical values for $m=2$ and $H a=100$ are $k_{\mathrm{c}}=44.03$ and $R e_{\mathrm{c}}=2590.83$ when the number of grids is 401 . Even in this quite high Hartmann case, the discrepancy between the two different systems of grid is small. For low values of Hartmann number, the critical Reynolds number depends significantly on the number of pole-pairs. However, this tendency is not observed for high values of Hartmann number. For each case of the number of pole-pairs, there is a Hartmann number that takes the minimum critical Reynolds number. These values are approximately 425, 505 and 591 for $m=1,2$ and 3, respectively. Figure 6 shows the critical Reynolds numbers and their corresponding axial wavenumbers as a function of Hartmann number for the three cases of magnetic pole-pairs. For the case of low Hartmann number $(\mathrm{Ha}<<1)$, the critical Reynolds number is proportional to $\mathrm{Ha}^{-2}$ irrespective of the value of $m$. Hence, the value of $H a^{2} R e_{\mathrm{C}}$ takes a constant value depending on the value of $m$. Both critical Reynolds number and critical wavenumber take almost the same value irrespective of $m$ for $\mathrm{Ha}>25$ since the basic core flow is synchronized with the phase velocity of the rotating magnetic field.

Figure 7 shows the neutral Reynolds number as a function of axial wavenumber for $\mathrm{Ha}=12$ and $m=2$ (Figure 7a) and each amplitude function at the critical point in which the axial wavenumber is 10.57 (Figure $7 \mathrm{~b}$ ). The region below the curve indicates the stable region while that above the curve is the unstable region and, therefore, the secondary flow takes place. For $\mathrm{Ha}=12$ and $m=2$, the Reynolds number takes its minimum value $\left(R e_{\mathrm{c}}=505.36\right)$ at $k=10.57$. On the right-hand side figure, normalization of amplitude functions is made so that $U=1$ at $R=0.9$. Figure 8 shows the visualization of the eigenfunction at the onset of instability for which the minimum critical Reynolds attained. These figures imply that there are almost three vortices aligned along the axial direction. It is quite interesting that the distribution of the Stokes' stream function is similar to that of the electric potential $\left(\varphi_{2}\right)$. 
Table 1. Critical Reynolds number obtained for the various cases changing number of pole-pairs and the Hartmann numbers. The number of grids is 201.

\begin{tabular}{ccccccc}
\hline \multirow{2}{*}{$\boldsymbol{H a}$} & \multicolumn{2}{c}{$\boldsymbol{m}=\mathbf{1}$} & \multicolumn{2}{c}{$\boldsymbol{m}=\mathbf{2}$} & \multicolumn{2}{c}{$\boldsymbol{m}=\mathbf{3}$} \\
\cline { 2 - 7 } & $\boldsymbol{R} \boldsymbol{e}_{\boldsymbol{c}}$ & $\boldsymbol{k}_{\boldsymbol{c}}$ & $\boldsymbol{R} \boldsymbol{e}_{\boldsymbol{c}}$ & $\boldsymbol{k}_{\boldsymbol{c}}$ & $\boldsymbol{R} \boldsymbol{e}_{\boldsymbol{c}}$ & $\boldsymbol{k}_{\boldsymbol{c}}$ \\
\hline 0.01 & $5.83401 \times 10^{7}$ & 6.5930 & $1.61817 \times 10^{8}$ & 7.8988 & $3.43488 \times 10^{8}$ & 9.0811 \\
0.1 & $5.83657 \times 10^{5}$ & 6.5934 & $1.61845 \times 10^{6}$ & 7.8990 & $3.43519 \times 10^{6}$ & 9.0813 \\
1 & 6094.99 & 6.6394 & $1.64646 \times 10^{4}$ & 7.9212 & $3.46678 \times 10^{4}$ & 9.0953 \\
2 & 1724.31 & 6.7755 & 4331.07 & 7.9879 & 8908.02 & 9.1375 \\
3 & 921.645 & 6.9925 & 2088.07 & 8.0979 & 4140.31 & 9.2076 \\
4 & 647.984 & 7.2776 & 1307.60 & 8.2491 & 2474.69 & 9.3049 \\
5 & 528.467 & 7.6162 & 951.267 & 8.4390 & 1707.10 & 9.4287 \\
6 & 470.275 & 7.9945 & 762.746 & 8.6646 & 1293.66 & 9.5781 \\
7 & 441.414 & 8.4007 & 654.126 & 8.9226 & 1048.01 & 9.7518 \\
8 & 428.429 & 8.8255 & 588.602 & 9.2091 & 892.271 & 9.9488 \\
9 & 424.848 & 9.2623 & 548.516 & 9.5206 & 789.215 & 10.168 \\
10 & 427.247 & 9.7064 & 524.505 & 9.8535 & 719.216 & 10.407 \\
11 & 433.675 & 10.155 & 511.207 & 10.204 & 671.111 & 10.665 \\
12 & 442.951 & 10.605 & 505.357 & 10.569 & 638.163 & 10.941 \\
13 & 454.327 & 11.055 & 504.868 & 10.947 & 616.096 & 11.233 \\
14 & 467.306 & 11.506 & 508.354 & 11.333 & 602.087 & 11.539 \\
15 & 481.549 & 11.955 & 514.862 & 11.727 & 594.199 & 11.858 \\
16 & 496.814 & 12.402 & 523.720 & 12.127 & 591.065 & 12.189 \\
17 & 512.926 & 12.848 & 534.443 & 12.531 & 591.695 & 12.529 \\
18 & 529.754 & 13.292 & 546.674 & 12.938 & 595.353 & 12.878 \\
19 & 547.199 & 13.734 & 560.147 & 13.347 & 601.484 & 13.235 \\
20 & 565.183 & 14.174 & 574.660 & 13.758 & 609.659 & 13.599 \\
25 & 661.439 & 16.340 & 658.372 & 15.817 & 671.639 & 15.482 \\
30 & 765.580 & 18.455 & 754.367 & 17.864 & 755.000 & 17.419 \\
50 & 1233.29 & 26.502 & 1203.84 & 25.783 & 1180.73 & 25.153 \\
100 & 2637.05 & 44.828 & 2585.30 & 44.042 & 2537.82 & 43.314 \\
\hline & & & & & &
\end{tabular}

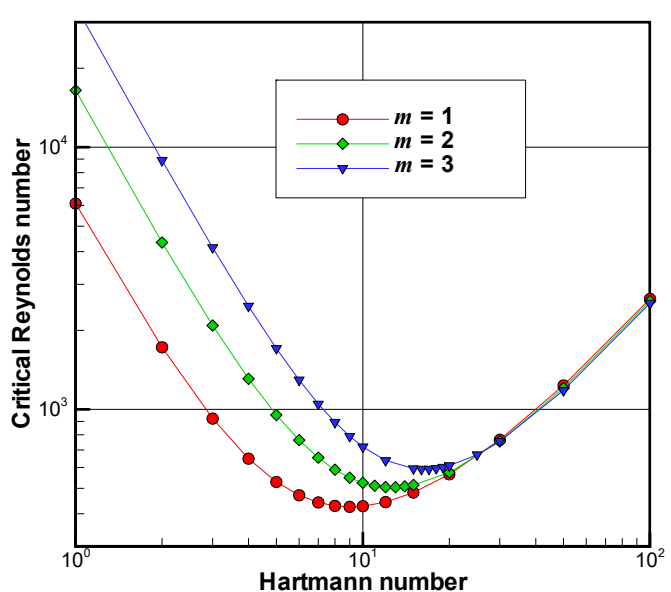

(a)

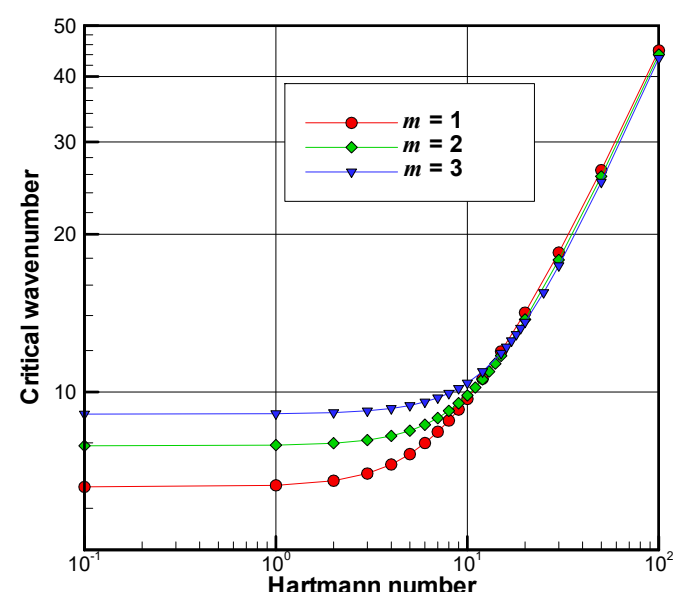

(b)

Figure 6. The critical Reynolds numbers and their corresponding axial wavenumbers as a function of the Hartmann number for the three cases of magnetic pole-pairs. (a) Critical Reynolds number; (b) critical wavenumber. 


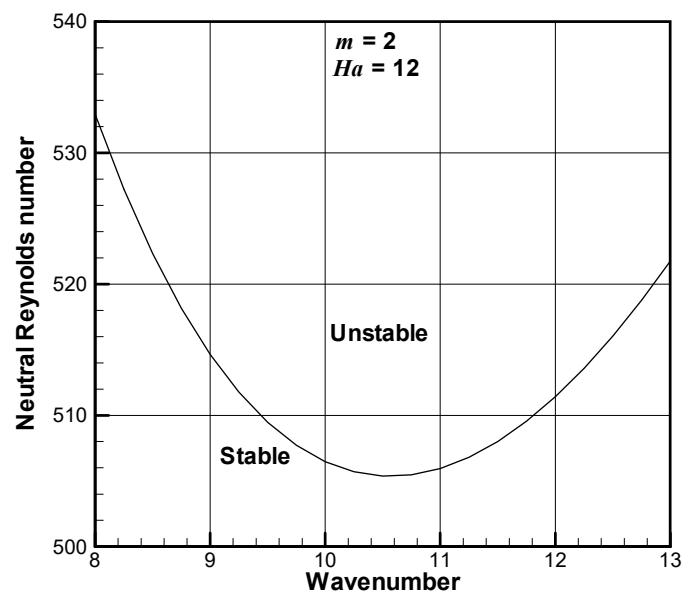

(a)

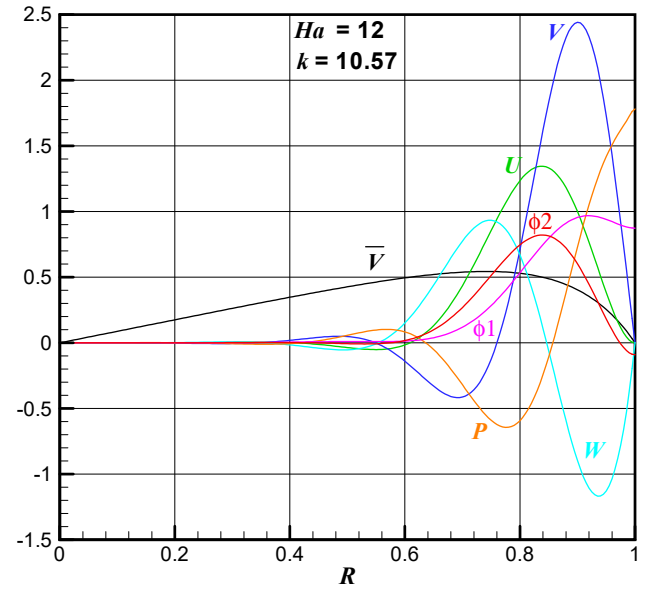

(b)

Figure 7. The neutral stability curve at $m=2$ and $H a=12$ shown in (a) and the amplitude functions for $m=2, k_{\mathrm{c}}=10.57$ and $H a=12$ shown in (b).

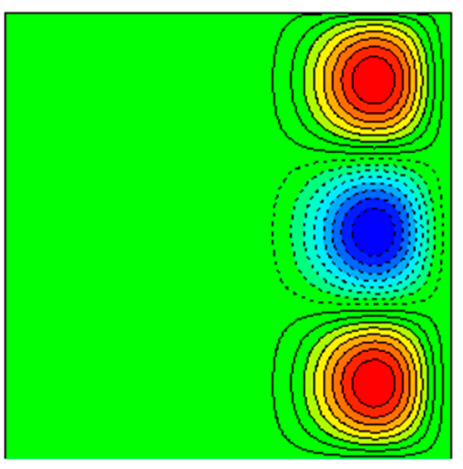

(a)

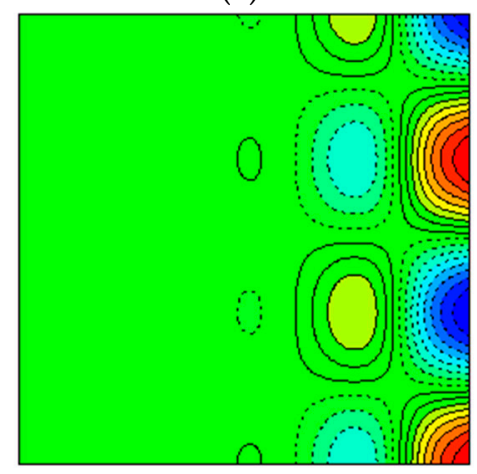

(c)

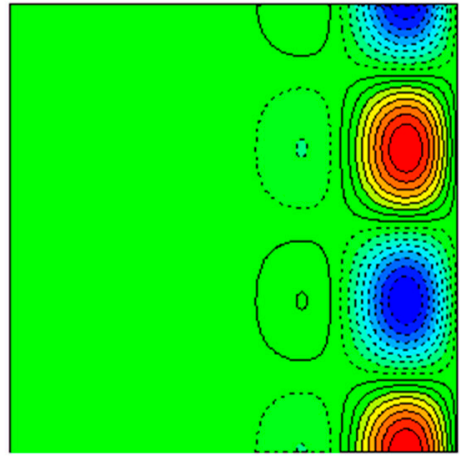

(b)

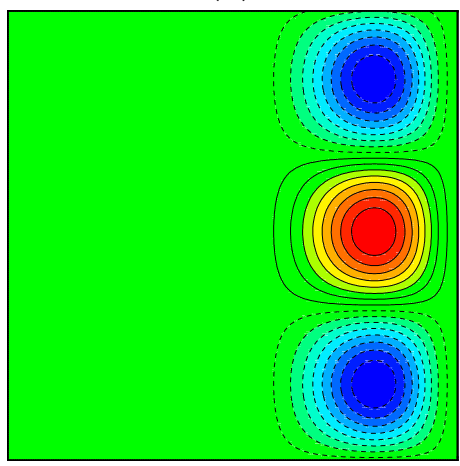

(d)

Figure 8. Visualization of the eigenfunctions at the onset of instability for $m=1, k_{\mathrm{c}}=9.262$ and $\mathrm{Ha}=9$. (a) Stream function; (b) azimuthal velocity; (c) pressure; (d) electric potential.

\section{Discussions}

\subsection{Magnetic Taylor Number}

Table 1 indicates that the value $H a^{2} R e$ in the limit of the small Hartmann number is 5834.0,16182, and 34349 for $m=1,2$, and 3, respectively. In this section, we consider the reason why these values approach each constant value in the limit of the small Hartmann number. First of all, the basic flow in the limit of the small Hartmann number is approximated as follows: 


$$
\bar{V}(R)=R-\frac{I_{1 / m}\left(H a R^{m} / \sqrt{2} m\right)}{I_{1 / m}(H a / \sqrt{2} m)} \cong \frac{H a^{2}}{8 m(m+1)} R\left(1-R^{2 m}\right),(\text { for } H a<<1)
$$

From Equation (58), the basic flow is proportional to the square of the Hartmann number. This solution can be obtained using Equation (59) in which the term of $\left(\mathrm{Ha}^{2} / 2\right) \mathrm{R}^{2(m-1)} \bar{V}$ in Equation (37) is neglected.

$$
\frac{d^{2} \bar{V}}{d R^{2}}+\frac{1}{R} \frac{d \bar{V}}{d R}-\frac{\bar{V}}{R^{2}}=-\frac{H a^{2}}{2} R^{2 m-1}
$$

This implies that Ohm's law, see Equation (6), is approximated using the following equation in which the induced electromotive force $(\boldsymbol{u} \times \boldsymbol{B})$ is dropped.

$$
j=\sigma \boldsymbol{E}=-\sigma \frac{\partial \boldsymbol{A}}{\partial t}=\left\{\frac{\sigma B_{0} a \omega}{m}\left(\frac{r}{a}\right)^{m} \sin (\omega t-m \theta)\right\} \boldsymbol{e}_{\boldsymbol{z}},(\text { for } H a<<1)
$$

Then, Equation (58) is substituted into Equations (51), (52) and (53), neglecting terms including $H a^{2}$, and the following simplified equations are obtained.

$$
\begin{gathered}
\left(D D_{*}-k^{2}\right) \tilde{U}-D \tilde{P}+T a_{m}\left(1-R^{2 m}\right) \tilde{V}=0 \\
\left(D D_{*}-k^{2}\right) \tilde{V}-T a_{m}\left[1-(m+1) R^{2 m}\right] \tilde{U}=0 \\
\left(D_{*} D-k^{2}\right) \tilde{W}-\mathrm{i} k \tilde{P}=0
\end{gathered}
$$

where, the magnetic Taylor number is defined as follows:

$$
T a_{m}=\frac{H a^{2} \operatorname{Re}}{4 m(m+1)}=\frac{\sigma B_{0}^{2} \Omega a^{4}}{4 m(m+1) \rho v^{2}}
$$

Eliminating the axial velocity by using Equations (50) and (63), and combining with Equation (61), we get the following equation without the expression of pressure:

$$
\left(D D_{*}-k^{2}\right)^{2} \tilde{U}=k^{2} T a_{m}\left(1-R^{2 m}\right) \tilde{V} .
$$

Richardson [2] used Equations (62) and (65) for $m=1$ in his analysis. According to his results, the critical magnetic Taylor number is 729.25 and the critical wavenumber is 6.59. The present numerical result for $m=1$ in the small limit of the small Hartmann number shown in Table 1 is consistent with this result.

\subsection{Validation of the Results}

For the low Hartmann number cases, the values of the shielding parameter are estimated by using Table 1. The magnetic Prandtl number for most liquid metals is in the order of $10^{-6}$ and we use this value for the estimation. When $H a=0.01, R_{\omega}=P r_{m} R e=58.34$ for $m=1$ and 343.5 for $m=3$. This implies that the fast rotating magnetic field hardly penetrates into the conducting fluid due to the significant skin effect. Therefore, these results would not be realized in a real system. When $\mathrm{Ha}=0.1$, $R_{\omega}=P r_{m} R e=0.5834$ for $m=1$ and 3.435 for $m=3$. In these cases, the magnetic field can penetrate the conducting fluid to some extent but the skin effect is still obvious. When $H a=1, R_{\omega}=P r_{m} R e=$ 0.006 for $m=1$ and 0.03 for $m=3$. In this case of Hartmann number, the skin effect can be neglected. The critical magnetic Taylor number is 761.9 for $m=1$ and 722.2 for $m=3$. These values are slightly larger than those of the low Hartmann number approximation discussed Section 4.1. This implies that the magnetic Taylor number is not useful for the determination of the onset of secondary flow but the rotational Reynolds number appears to be considered for each given Hartmann number. 
On the contrary, for high Hartmann number cases, the interaction parameter $N=H a^{2} / R e$, which is the ratio of the electromagnetic force to inertial force, becomes large. We should be careful about the value of $N$. For the case $N>>1$, it would be difficult to keep an axisymmetric circular flow. For example, for $m=1$ and $H a=100$, the value of $N$ is approximately 3.8, the assumption of axisymmetric flow in this study would not be valid. However, for $m=1$ and $H a=9$, the value of $N$ is approximately 0.19. In this case, the assumption of axisymmetric flow is valid since the basic azimuthal flow does not synchronize with the speed of the rotating magnetic field.

\subsection{Influence of Eelectric Potential}

In this subsection, we discuss the influence of the electric potential that appeared in Ohm's law, which governs the electric current density. Table 2 shows the comparison of the critical Reynolds number for various Hartmann numbers with and without taking the electric potential into account at $m=1$ [36]. When the Hartmann number is very small, since the term of the time-derivative of the vector potential is far superior to that of the induced electromotive force, the divergence-free condition for the electrical current density is automatically satisfied due to the employment of the Coulomb gauge. Therefore, the electric potential does not seem to be generated. As seen in Table 2 for the low values of Hartmann number, the difference of the critical Reynolds number and its corresponding wavenumber between the cases with and without the electric potential is negligible. However, for the high values of the Hartmann number, the difference between the two cases is significant. The electric potential makes this flow more unstable.

Table 2. The critical Reynolds number and the corresponding wavenumber for the various Hartmann numbers with and without taking the electric potential into account at $m=1$.

\begin{tabular}{ccccc}
\hline \multirow{2}{*}{$\boldsymbol{a} \boldsymbol{c}$} & \multicolumn{2}{c}{ With Electric Potential } & \multicolumn{2}{c}{ Without Electric Potential [36] } \\
\cline { 2 - 5 } & $\boldsymbol{R}_{\boldsymbol{c}}$ & $\boldsymbol{k}_{\boldsymbol{c}}$ & $\boldsymbol{R}_{\boldsymbol{c}}$ & $\boldsymbol{k}_{\boldsymbol{c}}$ \\
\hline 0.01 & $5.83401 \times 10^{7}$ & 6.5930 & $5.834 \times 10^{7}$ & 6.593 \\
0.1 & $5.83657 \times 10^{5}$ & 6.5934 & $5.836 \times 10^{7}$ & 6.594 \\
1 & 6094.99 & 6.6394 & 6116.6 & 6.649 \\
2 & 1724.31 & 6.7755 & 1747.8 & 6.813 \\
3 & 921.645 & 6.9925 & 948.25 & 7.074 \\
4 & 647.984 & 7.2776 & 678.73 & 7.416 \\
5 & 528.467 & 7.6162 & 564.31 & 7.821 \\
6 & 470.275 & 7.9945 & 512.03 & 8.274 \\
7 & 441.414 & 8.4007 & 489.80 & 8.759 \\
8 & 428.429 & 8.8255 & 484.08 & 9.268 \\
9 & 424.848 & 9.2623 & 488.32 & 9.792 \\
10 & 427.247 & 9.7064 & 499.08 & 10.33 \\
12 & 442.951 & 10.605 & 532.92 & 11.41 \\
15 & 481.549 & 11.955 & 601.97 & 13.06 \\
20 & 565.183 & 14.174 & 744.09 & 15.80 \\
30 & 765.580 & 18.455 & 1086.8 & 21.23 \\
50 & 1233.29 & 26.502 & 1924.4 & 31.87 \\
\hline
\end{tabular}

\section{Non-Linear Computations}

So far we have focused on the onset of secondary flow instability driven by the rotating magnetic field for an infinitely long cylinder case without taking the end effect into account. For the application of crystal growth of a semiconductor or other metal processes, it would be necessary to consider the end effect, as well as the aspect ratio of the height to the radius of the cylinder. Such discussion requires non-linear direct numerical computation. In this section, we focus on the influence of the boundary condition at the bottom and top walls on the supercritical flow for a finite cylinder case. 


\subsection{Periodic Condition}

In this section, a periodic boundary condition is considered to compare with the results obtained in the linear stability analyses. The boundary conditions employed for the periodic condition are shown in Equation (66).

$$
\left\{\begin{array}{l}
R=0: U=0, V=0, \partial W / \partial Z=0, \Phi_{1}=0, \Phi_{2}=0 \\
R=1: U=0, V=0, W=0, \partial \Phi_{1} / \partial R=0, \partial \Phi_{2} / \partial R=0 \\
Z=0,1: \partial U / \partial Z=0, \partial V / \partial Z=0, W=0, \Phi_{1}=0, \Phi_{2}=0
\end{array}\right.
$$

Figure 9 shows the growth rate of the maximum radial velocity for several values of the Reynolds number for the aspect ratio $A=1, m=1$ and $H a=9$ or 16 . The number of grids employed is $129 \times 129$ in the radial and axial directions, respectively. At the beginning of computation, each curve exhibits unpredictable behavior but it soon grows exponentially until the non-linear effect becomes significant. The critical Reynolds number obtained by the linear stability analysis for $m=1$ and $H a=9$ is approximately 425 , but the present non-linear analysis indicates that the growth rate at $R e=420$ is slightly positive. The same contradiction occurs at $\mathrm{Ha}=16$. This discrepancy between the linear and non-linear analyses may be due to the difference in the number of grids employed as well as the difference in the wavenumber. The critical wavenumber predicted by the linear analysis is about 9.26 , while the wavenumber by the non-linear analysis is equivalent to about 9.42 because there are three vortices aligned within the cylindrical enclosure for $A=1$. Figure 10 shows contour lines of the Stokes stream function and the electric potential $\Phi_{2}$ for the two different cases at the steady state that includes the non-linear effect. Since the value of Reynolds number is slightly higher than the critical condition, the fields of flow and electric potential are compared with Figure 8 for $m=1$ and $H a=9$. Due to the non-linear effect, which indicates that the perturbed flow is large enough to interact with the basic flow, the shape of the vortices is evidently distorted. However, the number of vortices is coincident between the linear and non-linear computations if the number of pole-pairs $m$ and the Hartmann number $\mathrm{Ha}$ are the same within this range of the Reynolds number Re.

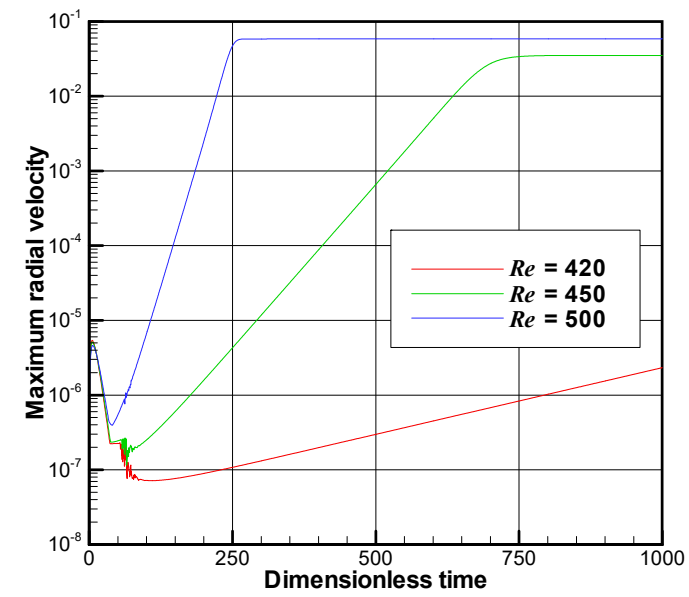

(a)

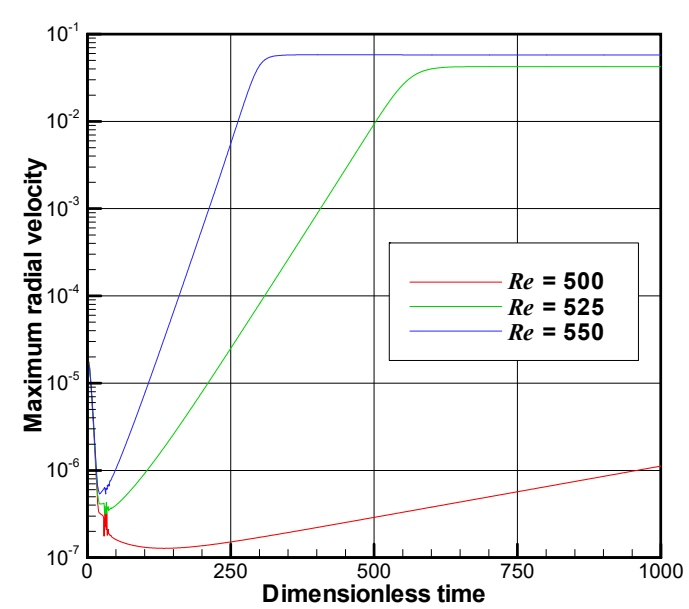

(b)

Figure 9. Growth rate of the maximum radial velocity for several values of the Reynolds number at $A=1$ and $m=1$. (a) $H a=9$; (b) $H a=16$. 


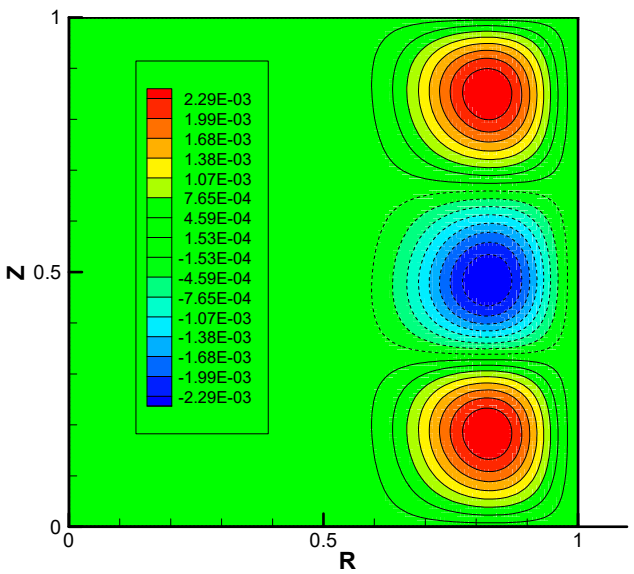

(a)

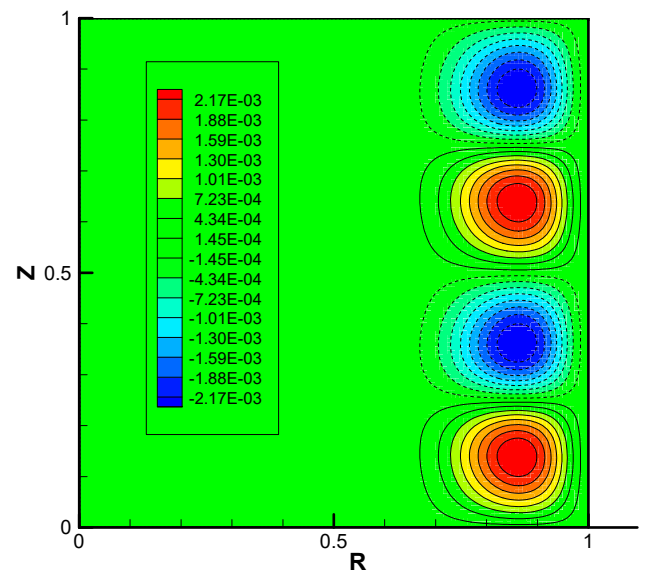

(c)

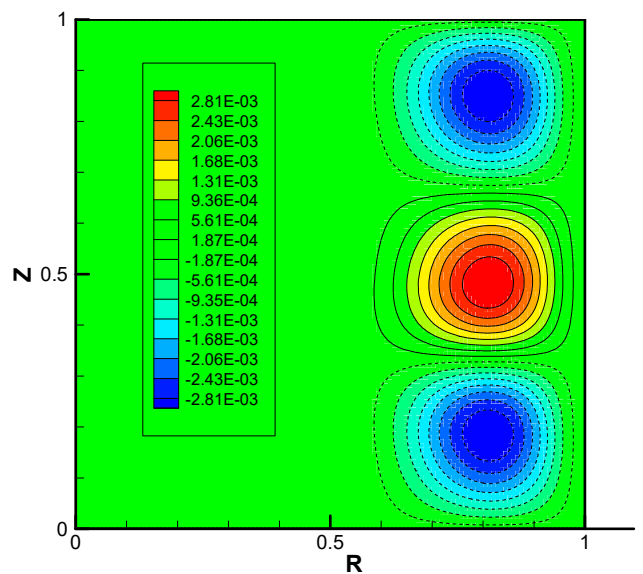

(b)

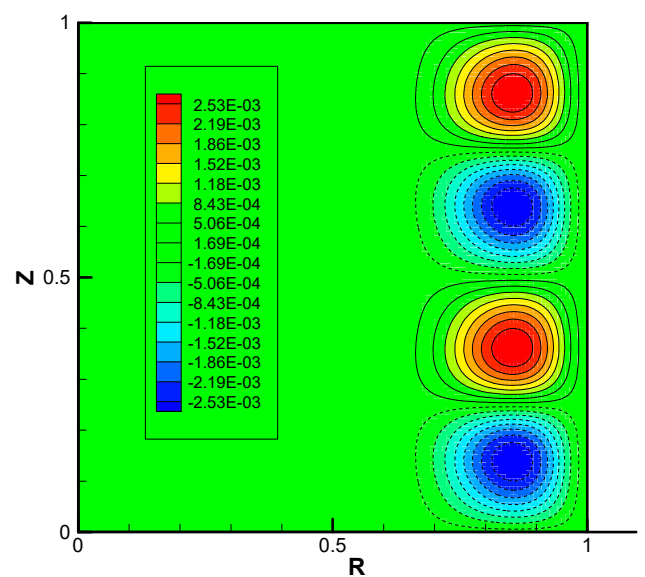

(d)

Figure 10. Contour line of the Stokes stream function and the electric potential at $A=1$ and $m=1$. (a) Stream function at $H a=9$ and $R e=450$; (b) electric potential at $H a=9$ and $R e=450$; (c) stream function at $H a=16$ and $R e=525$; (d) electric potential at $H a=16$ and $R e=525$.

\subsection{No-Slip Condition for $A=2$}

In this section, computational results with a no-slip condition at the top and bottom walls are discussed in order to compare with those with the slip condition. The effect of the aspect ratio would be crucial, but we would restrict the results only to $A=2$. The most striking difference from the slip condition is that the basic flow with the no-slip condition has always been the secondary flow. Figure 11 shows the contour maps for $m=1$ and $H a=8$ for the three different values of Reynolds number. The upper ones show the azimuthal velocity whereas the lower ones show the Stokes stream function. In the case of the no-slip condition at the top and bottom walls, the secondary flow has two large vortices when the Reynolds number is not very high. As the Reynolds number increases, the boundary layers formed in the vicinity of the side wall and that of the top and bottom walls become thin. Steady solutions were obtained until a Reynolds number of about 5000 for $\mathrm{Ha}=8$, beyond which the flow becomes unsteady. In the unsteady case, as shown in Figure 11c, a radially inward flow is observed at the mid-height of the side wall. However, this oscillatory flow is almost symmetrical with respect to $Z=1$ (mid-height). 

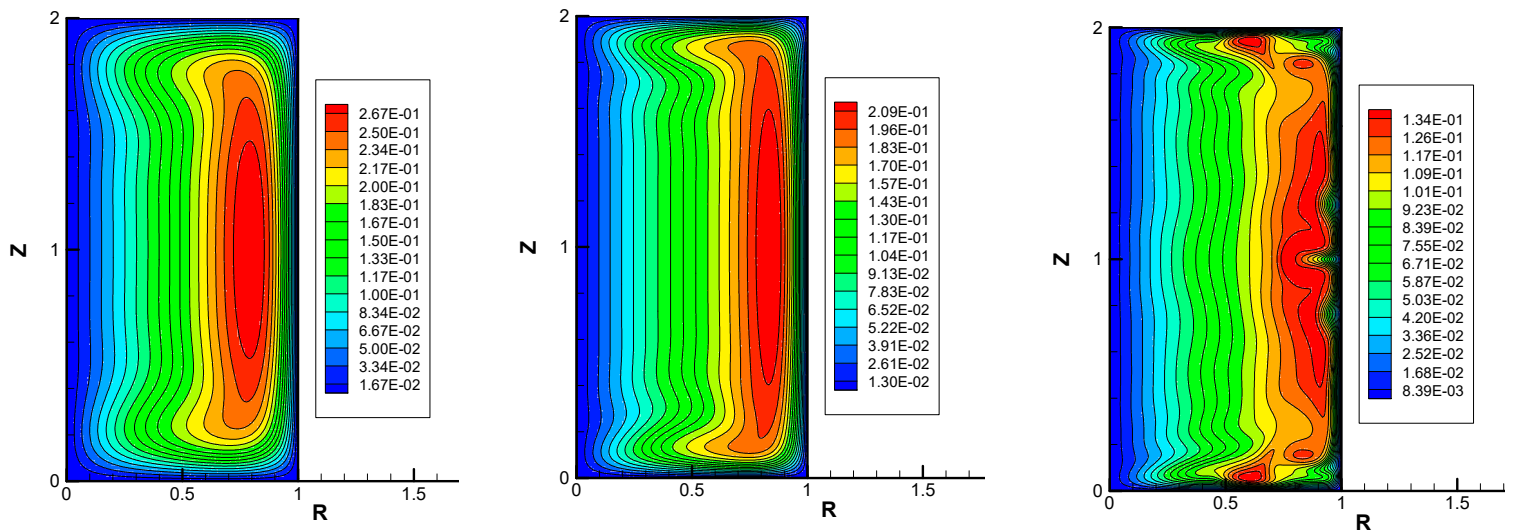

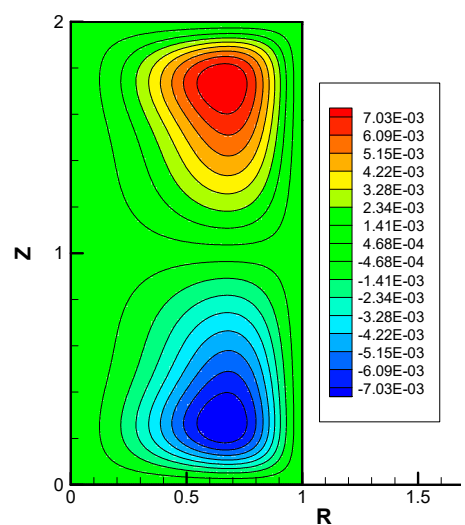

(a)

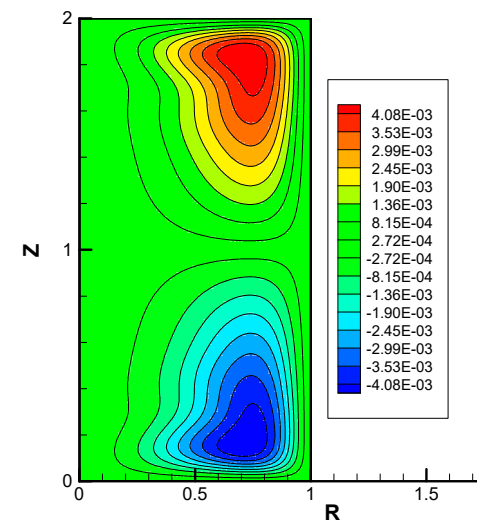

(b)

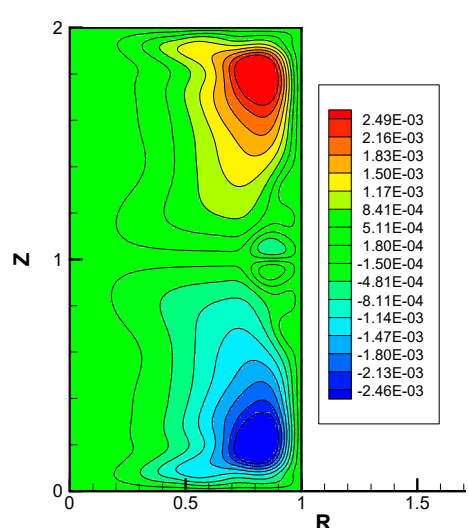

(c)

Figure 11. Contour maps of the azimuthal velocity (upper) and Stokes stream function (lower) for the three values of Reynolds number at $m=1$ and $H a=8$. The maps in (a) $R e=500$ and (b) $R e=2000$ indicate steady flow while that in (c) $R e=10000$ indicates an instantaneous snapshot because of the unsteady flow.

Figure 12 shows the contour lines of the azimuthal velocity for various combinations of the Hartmann number and the Reynolds number when the number of pole-pairs is 1 . As indicated in the previous figure, the flow transits from steady to unsteady as the Reynolds number increases but its value significantly depends on the Hartmann number. These Reynolds numbers indicate the approximate critical value above which the flow becomes oscillatory. The quasi-critical values are much larger than the critical values shown in Table 1 if the same Hartmann numbers are compared. Surprisingly, the azimuthal profiles are quite similar to each other, although the maximum azimuthal velocity, the value of which is written in each figure, is quite different. The common feature of this velocity profile is that there are oscillatory regions in the vicinity of the top and bottom walls. In this no-slip condition case, the top and bottom walls are stationary while the flow is mainly rotating in the azimuthal direction. Therefore, the Bödewadt boundary layer [37], which is known to exhibit an oscillatory profile, should be formed both in the vicinity of the top and bottom walls. Figure 13 shows the Bödewadt boundary layer obtained numerically by an additional computation assuming a similar solution. By comparing Figures 12 and 13, it is recognized that the Bödewadt boundary layer is approximately formed except in the region near the side wall where the adverse azimuthal velocity gradient is observed. 


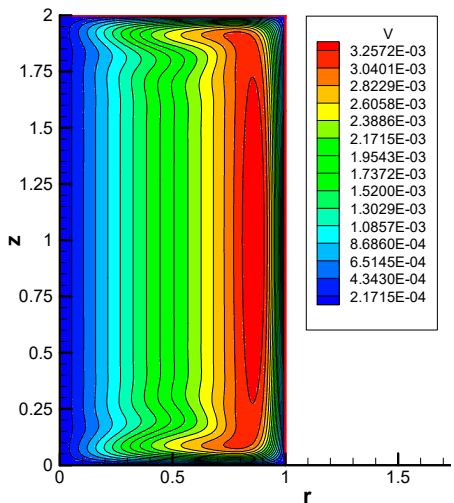

(a)

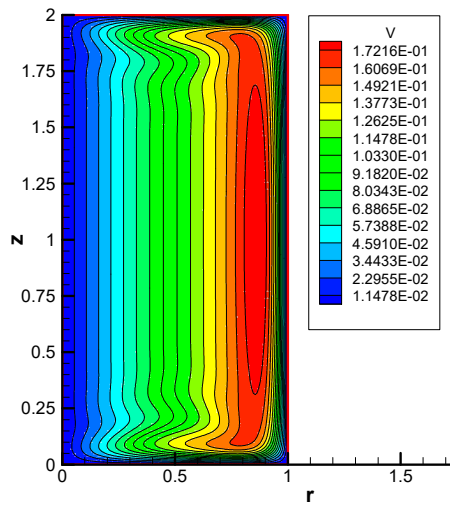

(d)

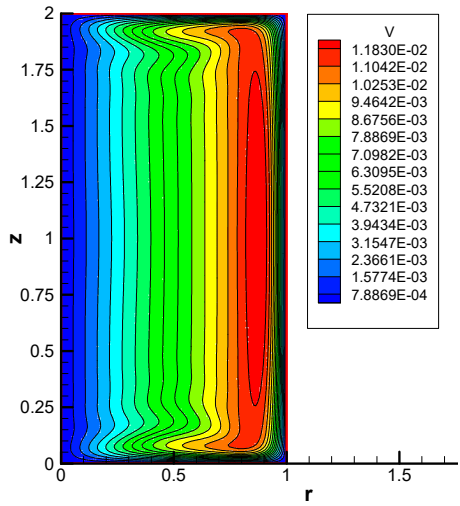

(b)

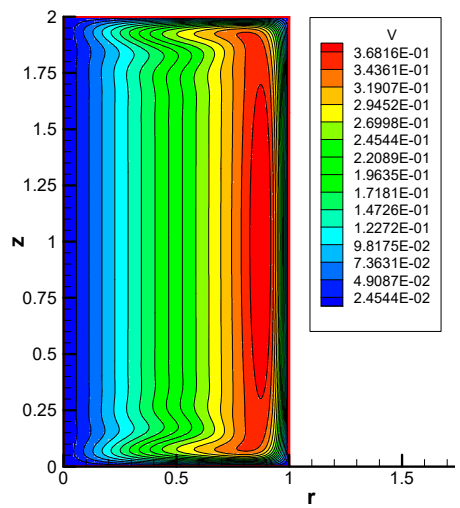

(e)

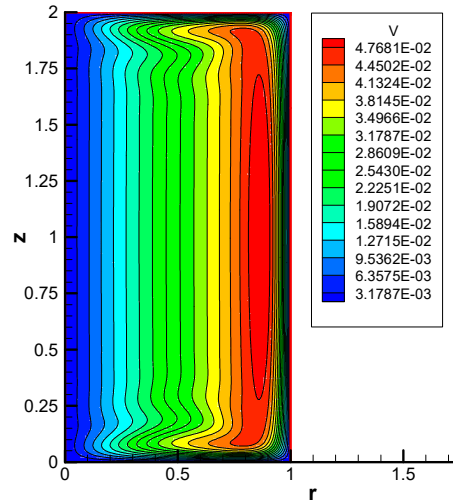

(c)

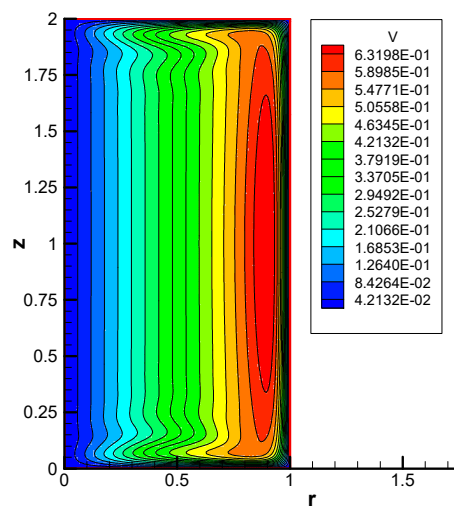

(f)

Figure 12. Contour maps of the azimuthal velocity for various combinations of the Hartmann number and the Reynolds number at $m=1$. (a) $H a=1, R e=300000$; (b) $H a=2, R e=100000$; (c) $H a=4$, $R e=22500 ;(\mathbf{d}) H a=8, R e=5000 ;(\mathbf{e}) H a=15, R e=3500 ;(\mathbf{f}) H a=30, R e=2410$.

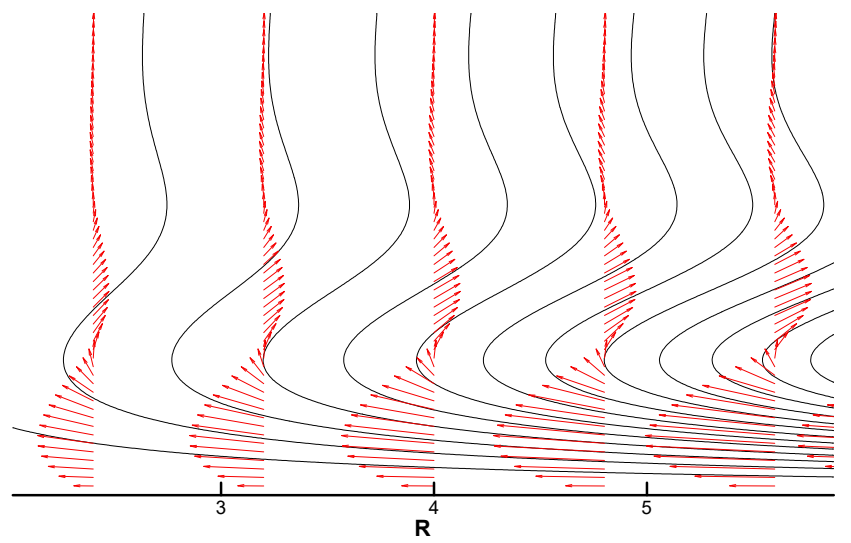

Figure 13. The meridional velocity (red vectors) and contour lines of Stokes stream function (black lines) in the Bödewadt boundary layer.

\section{Conclusions}

In this paper, the flow of the electrically conductive fluid in the cylindrical vessel driven by a multi-pole rotating magnetic field was discussed by using the linear stability analyses and non-linear direct numerical computations. The following conclusions were obtained.

1. For the cases in which the shielding parameter (magnetic Reynolds number) was much smaller than unity, the skin effect (induced magnetic field) was negligible. For such cases, the mathematical modeling of the rotating magnetic field with the arbitrary number of pole-pairs was derived. 
2. Decomposition of the electric potential into two parts allows us to derive the averaged electromagnetic force including potential gradient terms together with the assumption of the axisymmetric flow field.

3. For the infinitely long cylindrical vessel, the basic flow which has only an azimuthal component of velocity is not influenced by the electric potential. When the Hartmann number is very small, the influence of the number of pole-pairs on the basic flow is significant. When the Hartmann number is large, the dependency of the number of pole-pairs decreases and the angular velocity, except for at the boundary layer in the vicinity of the sidewall, synchronizes with the angular velocity of the rotating magnetic field.

4. For the low Hartmann number cases, the flow cannot follow the angular velocity of the rotating magnetic field. In such cases, the magnetic Taylor number determines the threshold of the onset of the secondary flow. This implies that both terms of the induced electromotive force and the potential gradient in Ohm's law can be neglected compared to the time-derivative of the vector potential. However, since it requires a very high angular velocity to generate the visible flow, the skin effect sometimes has to be taken into account.

5. For arbitrary cases of the Hartmann number, the Reynolds number based on the angular velocity of the rotating magnetic field determines the threshold of the onset of secondary flow instead of the magnetic Taylor number. In addition, there exists a certain Hartmann number that takes the minimum value of the critical Reynolds number, and its value depends on the number of the pole-pairs.

6. For the high Hartmann number cases, since the angular velocity of the magnetic field and the flow field are substantially synchronous, both terms of the induced electromotive force and the potential gradient in Ohm's law are significant. When the interaction parameter was much larger than unity, the electromagnetic force was superior to the inertia force, it appears that the axisymmetric circular flow might not be established. A non-axisymmetric time-dependent model may be required for such cases.

7. The non-linear direct computation suggests that the magnitude of the velocity component of the secondary flow increases exponentially as time evolves, and the growth of disturbances eventually stops after the non-linear effect becomes apparent and the velocity component becomes constant. Comparing the results between the linear stability analysis and non-linear direct computation, both results were in good agreement in terms of the critical value and the wave number.

8. The case with the no-slip boundary condition forms the Bödewadt boundary layer near the top and bottom walls and the basic flow includes the secondary flow. The stability of this case would depend on the aspect ratio; however, generally, it was much more stable than the flow with slip condition.

Author Contributions: Conceptualization, T.T.; writing—original draft preparation, T.T.; writing—review and editing, K.S.

Funding: This research received no external funding.

Conflicts of Interest: The authors declare no conflict of interest.

\section{Nomenclature}

a radius of cylinder $(\mathrm{m})$

A $\quad$ aspect ratio (-)

$A \quad$ vector potential $=A_{z} e_{z}(\mathrm{~T} \cdot \mathrm{m})$

$\boldsymbol{B} \quad$ magnetic induction $=B_{r} \boldsymbol{e}_{r}+B_{\theta} \boldsymbol{e}_{\theta}(\mathrm{T})$

$\boldsymbol{B}^{*} \quad$ dimensionless magnetic induction (-)

$B_{0} \quad$ absolute value of magnetic induction at $r=a(\mathrm{~T})$

$E \quad$ electric field $(\mathrm{V} / \mathrm{m})$

$\boldsymbol{e}_{r} \quad$ unit vector in radial direction (-)

$\boldsymbol{e}_{z} \quad$ unit vector in axial direction (-) 


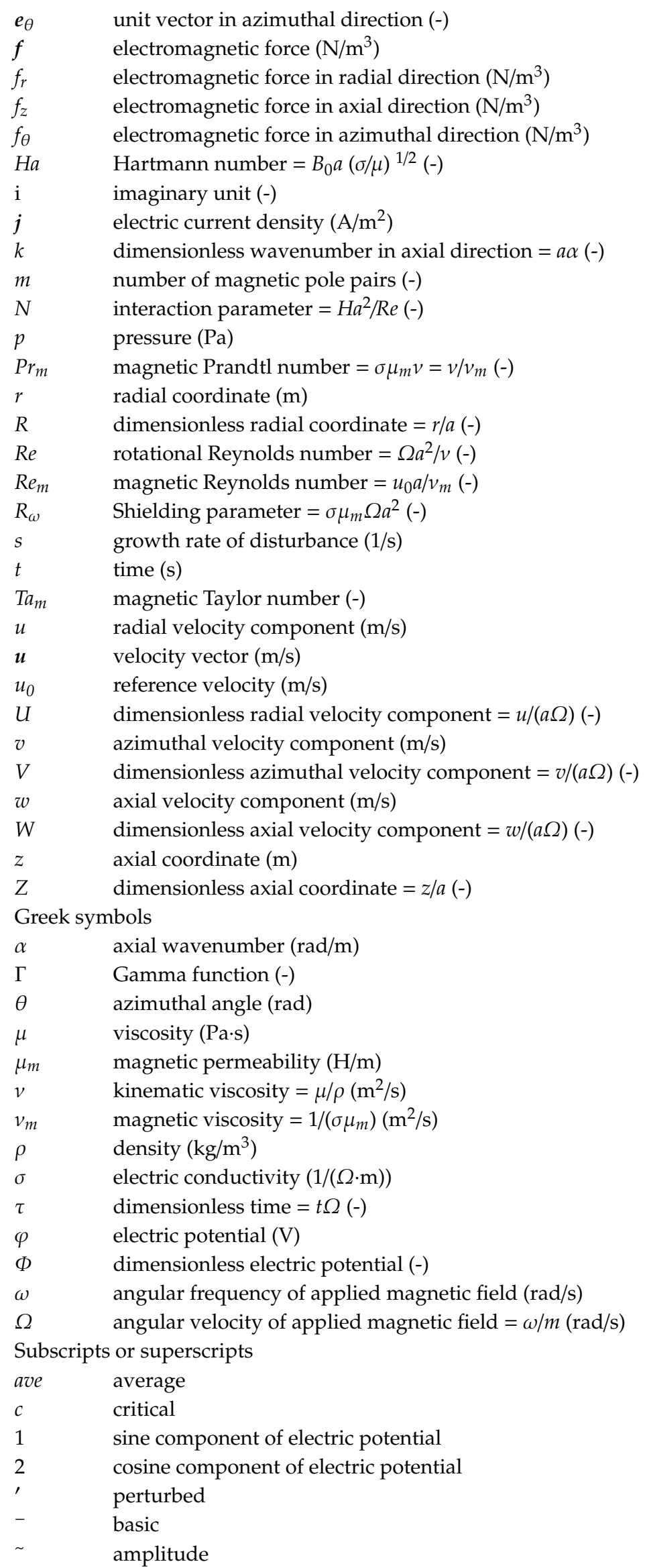




\section{References}

1. Moffatt, H.K. On fluid flow induced by a rotating magnetic field. J. Fluid Mech. 1965, 2, 521-528. [CrossRef]

2. Richardson, A.T. On the stability of a magnetically driven rotating fluid flow. J. Fluid Mech. 1974, 63, 593-605. [CrossRef]

3. Sneyd, A.D. Fluid flow induced by a rapidly alternating or rotating magnetic field. J. Fluid Mech. 1979, 92, 35-51. [CrossRef]

4. Davidson, P.A.; Hunt, J.C.R. Swirling recirculating flow in a liquid metal column generated by a rotating magnetic field. J. Fluid Mech. 1987, 185, 67-106. [CrossRef]

5. Davidson, P.A. Swirling flow in an axisymmetric cavity of arbitrary profile, driven by a rotating magnetic field. J. Fluid Mech. 1992, 245, 669-699. [CrossRef]

6. Barz, R.U.; Gerbeth, G.; Wunderwald, U.; Buhrig, E.; Gelfgat, Y.M. Modelling of the isothermal melt flow due to rotating magnetic fields in crystal growth. J. Cryst. Growth 1997, 180, 410-421. [CrossRef]

7. Ghaddar, C.K.; Lee, C.K.; Motakef, S.; Gillies, D.C. Numerical simulation of THM growth of CdTe in the presence of rotating magnetic fields (RMF). J. Cryst. Growth 1998, 205, 97-111. [CrossRef]

8. Mößner, R.; Gerbeth, G. Buoyant melt flows under the influence of steady and rotating magnetic fields. J. Cryst. Growth 1999, 197, 341-354. [CrossRef]

9. Ben Hadid, H.; Vaux, S.; Kaddeche, S. Three-dimensional flow transitions under a rotating magnetic field. J. Cryst. Growth 2001, 230, 57-62. [CrossRef]

10. Pätzold, O.; Grants, I.; Wunderwald, U.; Jenkner, K.; Cröll, A.; Gerbeth, G. Vertical gradient freeze growth of GaAs with a rotating magnetic field. J. Cryst. Growth 2002, 245, 237-246. [CrossRef]

11. Friedrich, J.; Lee, Y.S.; Fischer, B.; Kupfer, C.; Vizman, D.; Müller, G. Experimental and numerical study of Rayleigh-Bénard convection affected by a rotating magnetic field. Phys. Fluids 1999, 11, 853-861. [CrossRef]

12. Vizman, D.; Fischer, B.; Friedrich, J.; Müller, G. 3D numerical simulation of melt flow in the presence of a rotating magnetic field. Int. J. Numer. Methods Heat Fluid Flow 2000, 10, 366-384. [CrossRef]

13. Volz, M.P.; Mazuruk, K. Thermoconvective instability in a rotating magnetic field. Int. J. Heat Mass Transf. 1999, 42, 1037-1045. [CrossRef]

14. Volz, M.P.; Mazuruk, K. An experimental study of the influence of a rotating magnetic field on Rayleigh-Bénard convection. J. Fluid Mech. 2001, 444, 79-98. [CrossRef]

15. Grants, I.; Gerbeth, G. Stability of axially symmetric flow driven by a rotating magnetic field in a cylindrical cavity. J. Fluid Mech. 2001, 431, 407-426. [CrossRef]

16. Grants, I.; Gerbeth, G. Linear three-dimensional instability of a magnetically driven rotating flow. J. Fluid Mech. 2002, 463, 229-239. [CrossRef]

17. Grants, I.; Gerbeth, G. Experimental study of non-normal nonlinear transition to turbulence in a rotating magnetic field driven flow. Phys. Fluids 2003, 15, 2803-2809. [CrossRef]

18. Martin Witkowski, L.; Marty, P. Effect of a rotating magnetic field of arbitrary frequency on a liquid metal column. Eur. J. Mech. B Fluids 1998, 17, 239-254. [CrossRef]

19. Martin Witkowski, L.; Walker, J.S.; Marty, P. Nonaxisymmetric flow in a finite-length cylinder with a rotating magnetic field. Phys. Fluids 1999, 11, 1821-1826. [CrossRef]

20. Martin Witkowski, L.; Marty, P.; Walker, J.S. Liquid-metal flow in a finite-length cylinder with a high-frequency rotating magnetic field. J. Fluid Mech. 2001, 436, 131-143. [CrossRef]

21. Walker, J.S.; Martin Witkowski, L.; Houchens, B.C. Effects of a rotating magnetic field on the thermocapillary instability in the floating zone process. J. Cryst. Growth 2003, 252, 413-423. [CrossRef]

22. Walker, J.S.; Martin Witkowski, L. Linear stability analysis for a rotating cylinder with a rotating magnetic field. Phys. Fluids 2004, 16, 2294-2299. [CrossRef]

23. Feonychev, A.I.; Bodareva, N.V. Effect of a rotating magnetic field on convection stability and crystal growth in zero gravity and on the ground. J. Eng. Phys. Thermophys. 2004, 77, 731-742. [CrossRef]

24. Nikrityuk, P.A.; Eckert, K.; Grudmann, R. Numerical study of a laminar melt flow driven by a rotating magnetic field in enclosed cylinders with different aspect ratios. Acta Mech. 2006, 186, 17-35. [CrossRef]

25. Lyubimov, D.V.; Burnysheva, A.V.; Ben Hadid, H.; Lyubimova, T.P.; Henry, D. Rotating magnetic field effect on convection and its stability in a horizontal cylinder subjected to a longitudinal temperature gradient. J. Fluid Mech. 2010, 664, 108-137. [CrossRef] 
26. Ando, T.; Ueno, K.; Taniguchi, S.; Takagi, T. Visual system experiment of MHD pump using rotating twisted magnetic field applicable to high-temperature molten metals. ISIJ Int. 2003, 43, 849-854. [CrossRef]

27. Ando, T.; Ueno, K.; Taniguchi, S.; Takagi, T. Induction pump for high-temperature molten metals using rotating twisted magnetic field: Thrust measurement experiments with solid conductors. IEEE Trans. Magn. 2004, 38, 1789-1796. [CrossRef]

28. Ando, T.; Ueno, K.; Taniguchi, S.; Takagi, T. Induction pump for high-temperature molten metals using rotating twisted magnetic field: Molten gallium experiment. IEEE Trans. Magn. 2004, 40, 1846-1857. [CrossRef]

29. Ando, T.; Ueno, K.; Sawada, K. Numerical simulation of molten metal flow produced by induction MHD pump using rotating twisted magnetic field. JSME Int. J. Ser. B 2005, 48, 508-516. [CrossRef]

30. Ueno, K.; Ando, T. Theoretical study of induction pump for molten metal using rotating twisted magnetic field. IEEE Trans. Magn. 2012, 48, 1200-1211. [CrossRef]

31. Priede, J. Theoretical Study of a Flow in an Axisymmetric Cavity of Finite Length, Driven by A Rotating Magnetic Field. Ph.D. Thesis, Latvian Academy of Sciences Institute of Physics, Salaspils, Latvian, 1993.

32. Priede, J.; Gelfgat, Y.M. Mathematical model of the mean electromagnetic force induced by a rotating magnetic field a liquid column of a finite length. Magnetohydrodynamics 1996, 32, 272-280.

33. Priede, J.; Gerbeth, G. A model of low-frequency rotating magnetic field with a weak axial gradient. Magnetohydrodynamics 2005, 41, 189-198.

34. Moreau, R. Magnetohydrodynamics; Kluwer Academic Publishers: Dordrecht, The Netherlands, 1990.

35. Hirt, C.W.; Nichols, B.D.; Romero, N.C. SOLA: A Numerical Solution Algorithm for Transient Fluid Flows; Los Alamos Scientific Lab., N. Mex.: Los Alamos, NM, USA, 1975.

36. Tagawa, T.; Egashira, R. Fluid flow of a liquid metal in a cylinder driven by a rotating magnetic field. Trans. Jpn. Soc. Mech. Eng. Part B 2012, 78, 1680-1695. [CrossRef]

37. Bödewadt, U.T. Die Drehströmung über festem Grunde. ZAMM 1940, 20, 241-245. [CrossRef]

(C) 2019 by the authors. Licensee MDPI, Basel, Switzerland. This article is an open access article distributed under the terms and conditions of the Creative Commons Attribution (CC BY) license (http://creativecommons.org/licenses/by/4.0/). 